\title{
High performance ambipolar organic mixed ionic-electronic conductor for adaptive logic circuits and neuromorphic electronics
}

Authors: Yanxi Zhang ${ }^{1 * \#}$, Eveline R. W. van Doremaele ${ }^{1 \#}$, Gang Ye $e^{2,3^{*}}$, Tim Stevens ${ }^{1}$, Jun Song ${ }^{2^{*}}$, Ryan C. Chiechi ${ }^{3,4}$, Yoeri van de Burgt ${ }^{1^{*}}$

\author{
Affiliations: \\ ${ }^{1}$ Microsystems, Department of Mechanical Engineering and Institute for Complex Molecular \\ Systems, Eindhoven University of Technology, 5600 MB, Eindhoven, The Netherlands. \\ ${ }^{2}$ Center for Biomedical Optics and Photonics (CBOP) \& College of Physics and \\ Optoelectronic Engineering, Key Laboratory of Optoelectronic Devices and Systems, \\ Shenzhen University, Shenzhen 518060, P. R. China \\ ${ }^{3}$ Stratingh Institute for Chemistry and Zernike Institute for Advanced Materials, University of \\ Groningen, 9747 AG Groningen, The Netherlands \\ ${ }^{4}$ Present address: Department of Chemistry, North Carolina State University, Raleigh, North \\ Carolina 27695-8204, United States \\ \#These authors contributed equally.
"Correspondence to: y.zhang9@tue.nl, g.ye0612@szu.edu.cn, songjun@szu.edu.cn,
y.b.v.d.burgt@tue.nl
}

Organic mixed ionic-electronic conductors (OMIECs) are central to bioelectronic applications such as biosensors ${ }^{1}$, health monitoring devices and neural interfaces ${ }^{2}$, and have facilitated efficient next-generation brain-inspired computing ${ }^{3}$ and biohybrid systems ${ }^{4}$. Most OMIECs are hole-conducting (p-type) materials, while complimentary logic circuits and various biosensors require electron-conducting (n-type) materials too ${ }^{5,6}$. Here we show an ambipolar mixed ionic-electronic polymer that achieves high on/off ratios with high ambient $p$ and $n$ - type stability. We highlight the versatility of the material by demonstrating its use as a neuromorphic memory element, an adaptable ambipolar complementary logic inverter, and a neurotransmitter sensor. The ambipolar operation of this material allows for straightforward monolithic fabrication and integration, and opens a route towards more sophisticated complex logic and adaptive circuits.

The success of many organic bioelectronic applications results from the reduced interface impedance and the extraordinarily high electrochemical transconductance in OMIECs ${ }^{7,8}$. These and other unique properties such as biocompatibility, low voltage operation, simple fabrication processing, stretchable and flexible materials, and the extensive capabilities in tailoring molecular structures and functions via chemical synthesis has opened a wide variety of possible bioelectronic applications such as neural probes ${ }^{7}$ and sensors for ions ${ }^{9}$, metabolites $^{5}$, bacteria ${ }^{10}$, and viruses ${ }^{11}$. Furthermore, the huge dynamic range and electrochemical doping nature of these polymers has produced tuneable analogue neuromorphic devices ${ }^{3,12}$ with high stability ${ }^{13}$ resulting in proof-of-principle circuits ${ }^{14,15}$ as well as biohybrid systems directly modulated by neurotransmitters secreted locally ${ }^{4}$. Current applications are often based on the commercially available poly(3,4-ethylenedioxythiophene): polystyrene sulfonate (PEDOT:PSS), which is a p-type (hole) conductive polymer that operates in depletion-mode. This means that the material is conductive and can be turned off by applying a gate potential. Enhancement-mode p-type devices exist, notable by design ${ }^{16}$ or by dedoping of PEDOT:PSS with small molecule amines ${ }^{17}$. However, in order to form logic gates, complementary circuits, and energy storage devices, a combination of $p$ and n-type materials is required ${ }^{6}$. Besides, n-type OMIECs are essential for detecting metabolites like glucose and lactase in enhancement mode organic electrochemical transistors (OECTs) $)^{5}$. 
Despite various efforts to explore n-type materials, to date the amount and the performance of $n$-type OMIECs is still inferior compared to its $p$-type counterpart. The key issue is that (doped) n-type materials in general are extremely unstable when exposed to air, combined with the challenge of achieving high electron mobility. Another challenge is to obtain OMIECs that can be doped by both cations and anions and can conduct both holes and electrons. One strategy to design n-type conjugated polymers is copolymerizing an electron deficient moiety (acceptor), such as naphthalenediimide (NDI), with an electron rich moiety (donor) like thiophene. These donor-acceptor (D-A) polymers are often modified with hydrophilic ethylene glycol (EG) type side chains for applications as aqueous electrolyte gated transistors, glucose sensing, and biofuel applications ${ }^{18}$. The EG chains support highly efficient electrochemical doping within the whole volume and enables low voltage $(<1 \mathrm{~V})$ operation, which is essential when using an aqueous electrolyte. In order to properly operate stable devices in water, a LUMO level below $-4 \mathrm{eV}$ is required for $\mathrm{n}$-type polymers to avoid side reactions with water and oxygen ${ }^{19}$. High performance n-type OMIECs require balancing the ions and electron mobility, while maintaining unperturbed crystalline structures upon electrochemical doping ${ }^{20}$. It has been reported that embedding $\mathrm{sp}^{2}-\mathrm{N}$ into polymer backbones results in a more planar structure, enhancing the $\pi-\pi$ stacking, and increasing the electron mobility ${ }^{21,22}$. Capitalizing on these properties, the material we present here is an NDI-bithiozale D-A copolymer (referred to as P-3O, shown in Fig. 1a) functionalized with EG side chains, for stable operation both in aqueous environments as well as with a solid electrolyte.

An all-solid-state environment is desirable in applications associated with electronic circuitry, logic circuits, and neuromorphic computing, as the operation of circuits in aqueous environments can lead to interference with the surrounding electronics as well as neighboring devices. From a technological point of view the use of liquid electrolytes limits the high-density integration of devices. ${ }^{23}$ The solid electrolyte consists of an ionic liquid (1-ethyl-3methylimidazolium bis(trifluorosulfonyl)imide, EMIM:TFSI) within a polymer matrix (poly(vinylidene fluoride-co-hexafluoropropylene), PVDF-HFP) ${ }^{24}$. Figure $1 \mathrm{a}$ shows the architecture of the solid electrolyte-gated device. The devices can be designed with a side gate which simplifies the fabrication by avoiding a complex layer-by-layer process to construct a stacked structure. Figure $1 \mathrm{~b}$ and $1 \mathrm{c}$ give the output and transfer characteristics of the P-30 OECT showing a near-symmetric ambipolar electrical behaviour when operated in ambient environment with a solid gel-electrolyte. The polymer achieves an on/off ratio of $10^{4}$ and shows stable operation for more than 20,000 cycles for both p- and n-type in ambient conditions, see Figure $1 \mathrm{~d}$ and $1 \mathrm{e}$. This all-solid-state device allows for straightforward fabrication and facile integration of large circuitry such as an array architecture often used in neuromorphic computing or integrated adaptable electronic circuits. 
a)

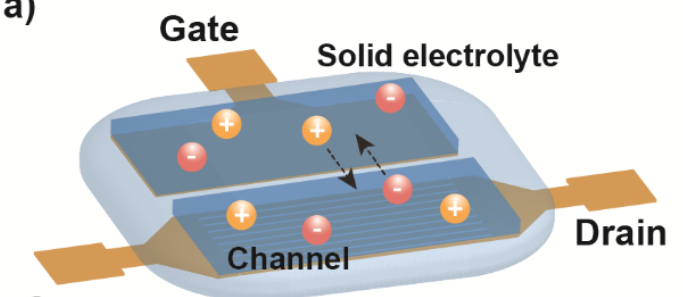

Source
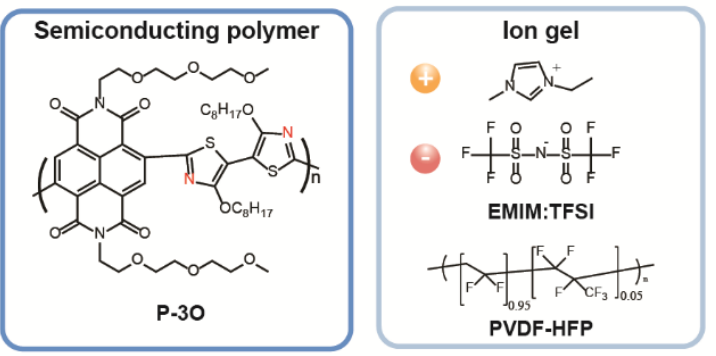

d)

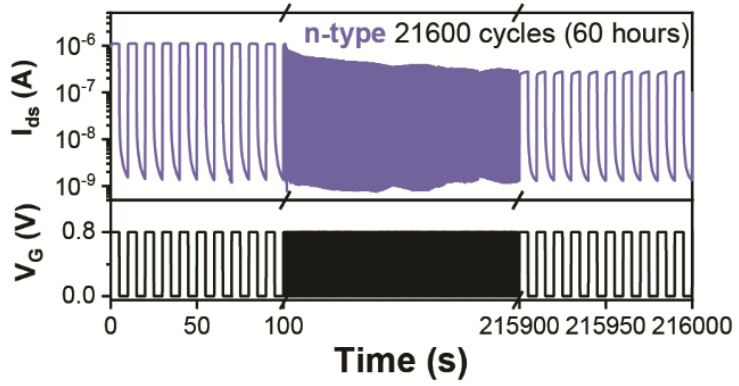

b)
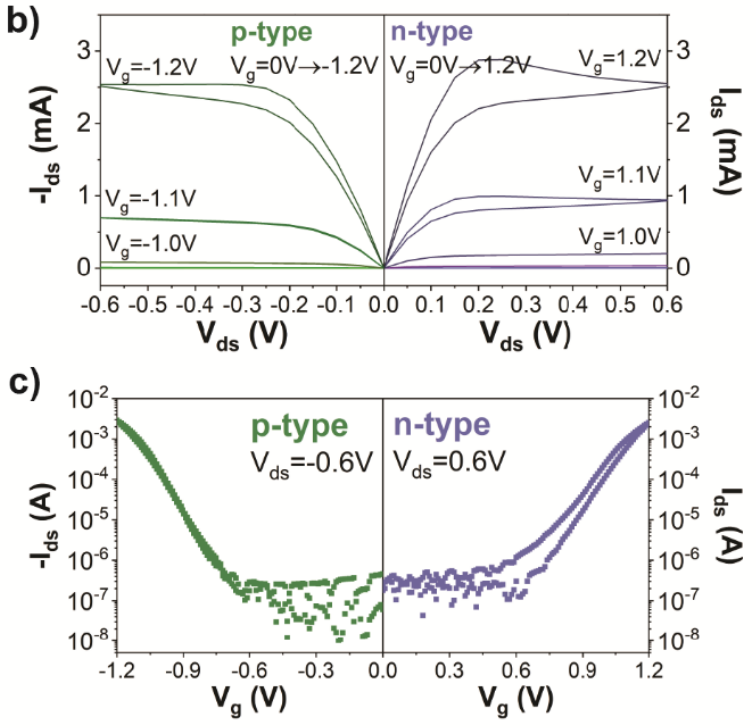

e)

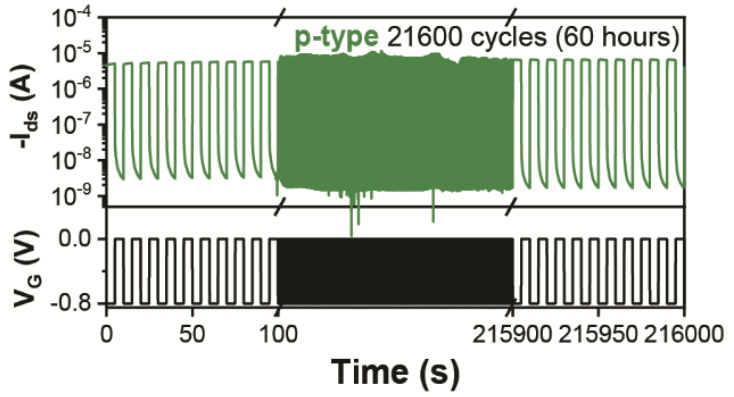

Figure 1| Solid-state ion-gated transistor. a) Schematic of the ion gel side-gated organic electrochemical transistor. Chemical structures of the conjugated D-A polymer P-3O and ion gel. b) Ambipolar output curve. c) Ambipolar semi-log transfer curve carried out in ambient environment. d) n-type and e) p-type operational stability measurement of the ion gel gated transistor. The drain current $\left(I_{d s}\right)$ was monitored when applying a $5 \mathrm{~s}$ gate voltage pulse $\left|V_{\mathrm{G}}\right|=0.8 \mathrm{~V}$ (interval time of $5 \mathrm{~s}$ ) for 21600 pulse cycles $\left(\left|V_{d s}\right|=0.1 \mathrm{~V}\right)$.

We demonstrate this capability by fabricating an adaptive memory device, an electrochemical random-access memory (EC-RAM) based on P-3O. The EC-RAMs adopt a similar configuration as solid-state electrochemical transistors and maintains non-volatile memory by employing an open gate circuit in combination with a current limiting resistor (see Fig. 2a). The conductance can be randomly accessed over a large range and in an analog fashion, allowing these devices to act as synaptic weights in hardware artificial neural networks, enabling parallel computation ${ }^{15}$ and increasing the energy efficiency. Organic EC-RAMs are able to operate at low voltage and with high write speeds ( $20 \mathrm{~ns}$ pulse) ${ }^{25}$ The P-3O EC-RAMs function in n-type mode (positive read and write voltage) and can access over $2^{5}$ conductance states across two orders of magnitude, in linear fashion, and with high state retention (see Fig. 2). Figure $2 \mathrm{~b}$ shows a state retention of 5 minutes in nitrogen atmosphere. Due to the presence of oxygen and water, encapsulation is needed for operating in ambient conditions (see Fig. S19). The state retention can be further improved by optimising the device geometry and purifying the ion gel. The n-type P-3O-based EC-RAM exhibits comparable performance to $p$ type polymer based EC-RAMs such as PEDOT:PSS ${ }^{13}, \mathrm{p}(\mathrm{g} 2 \mathrm{~T}-\mathrm{TT})^{25}$, and $\mathrm{P} 3 \mathrm{HT}^{26}$. Even though the P-3O polymer is ambipolar, the p-type mode (negative read and write voltage) ECRAM decays faster even in nitrogen atmosphere (see Fig. S20) which might indicate that the energetic barrier for back diffusion of the anions ( $\mathrm{TFSI}^{-}$) is low ${ }^{27}$. The n-type EC-RAMs can be further integrated in large crossbar arrays to emulate the synaptic weights in an artificial neural network. We simulate the behaviour of such an array, in its performance to recognize and 
classify handwritten digits (see Supplementary Information). The linear and symmetric behaviour of potentiation and depression of the conductance (see Fig. 2e) results in efficient training and inference of the neural network. Figure $2 \mathrm{f}$ shows that for both the small and large handwritten digits dataset the accuracy of training an EC-RAM is comparable to the ideal numeric accuracy, which is the theoretical limit for this algorithm.

a)

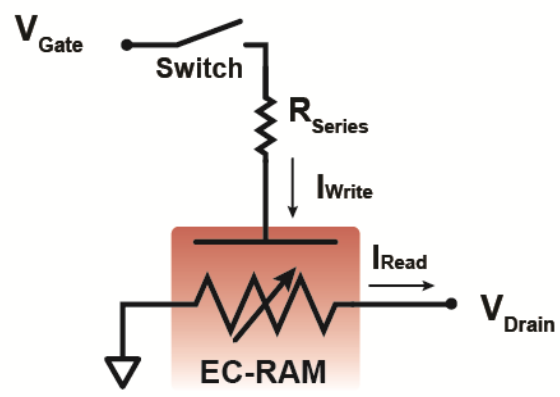

c)

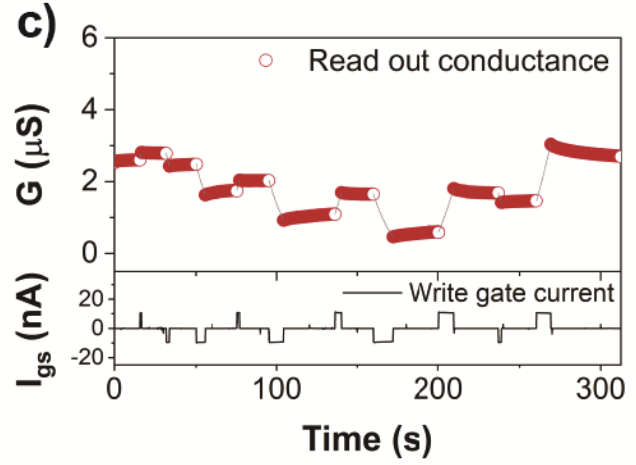

e)

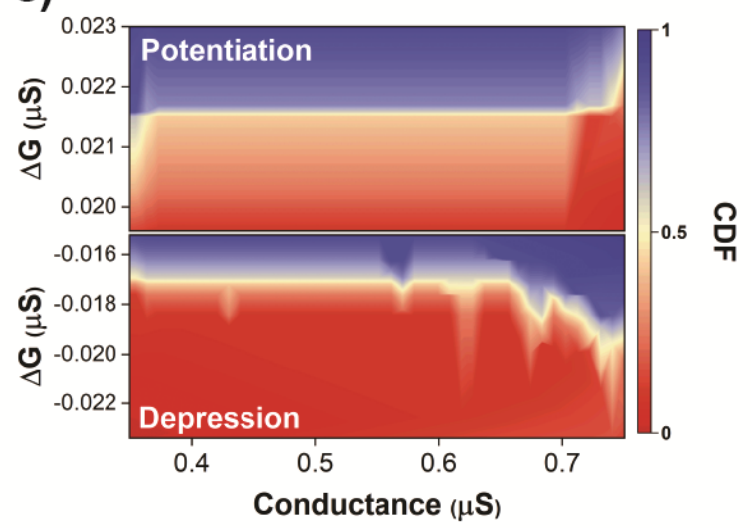

b)

d)

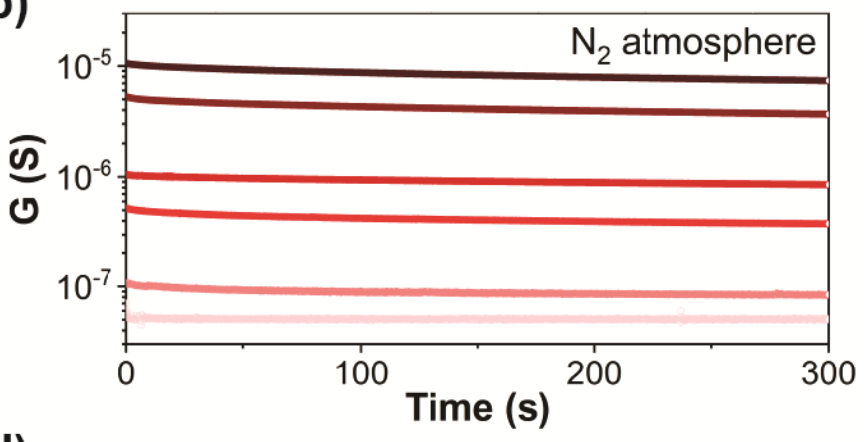

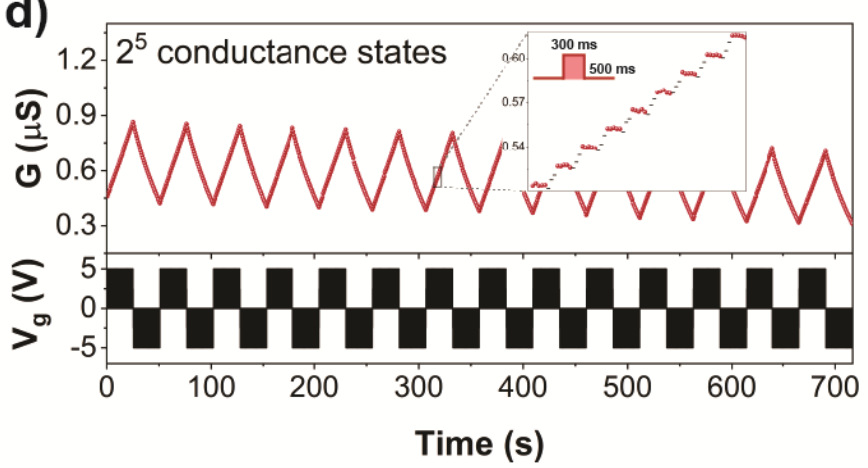

f)
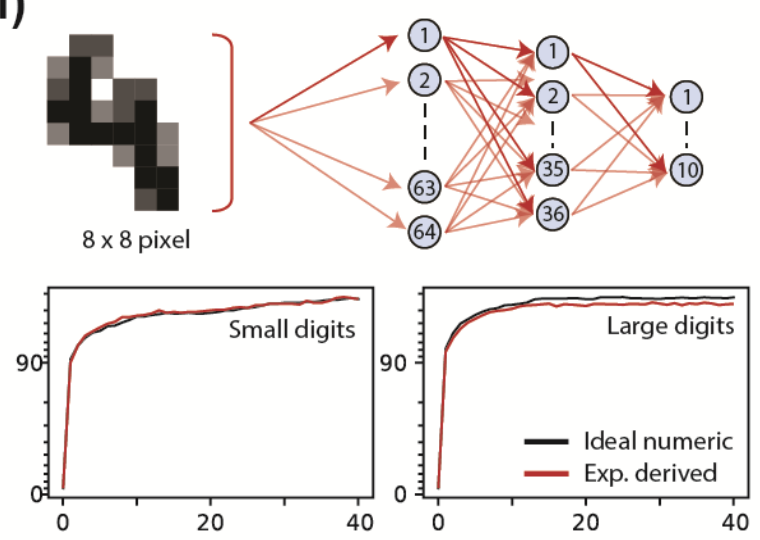

Figure 2| Solid-state ion-gated EC-RAM. a) Schematic of the EC-RAM with a resistor in series to limit the gate current, used to access different conductance states. b) State retention in a $\mathrm{N}_{2}$ environment for 5 minutes at different conductance states over two orders of magnitude. c) Behaviour over time when tuned to different discrete conductance states. d) 14 cycles of 32 conductance states showing linear potentiation and depression (pulse width 300 ms and delay 500 ms with a $1 \mathrm{GOhm}$ resistor). e) Heat map of the change in conductance across the conductance range for both potentiation and depression with the colour representing the cumulative distribution function (CDF) at each conductance state. f) Training results of the simulation showing the accuracy of the ideal numerical and experimentally derived neural network for 40 epochs classifying 8x8 pixel (small) and 28x28 pixel (large) handwritten digits ${ }^{28}$.

Due to the excellent performance of the P-30 ambipolar polymer that spans across multiple different functional areas, complex and integrated circuits can be realized while remaining a straightforward monolithic fabrication process. Since both the $\mathrm{p}$ - and $\mathrm{n}$-type transfer curves 
have a near-identical behaviour (shown in Fig. 1c), we can fabricate an inverter, a fundamental building block of electronic circuits (see Fig. 3). Inverters are used in both digital circuits, as logic gates, and in analogue circuits as voltage amplifiers since it inverts the applied input voltage $\left(V_{\text {in }}\right)$ from a high to low voltage and vice versa (see Fig. 3a and $b$ ). At the transition region it shows a high gain which makes an organic inverter find relevant and wide applications in several fields, including for example printed electronics, imperceptible and wearable electronics, sensors and bioelectronics ${ }^{29-31}$. Figure $3 a$ shows the performance of the solidstate ambipolar inverter with a gain of 12 at $\mathrm{V}_{\mathrm{DD}}=0.8 \mathrm{~V}$, along with Figure $3 \mathrm{~b}$ presenting the logic NOT gate operation where a high input results in a low output voltage. To highlight opportunities for more advanced bioelectronic applications as well as future personalized and adaptive systems operating locally, we combine the ambipolar inverter with the EC-RAM in order to realize an adaptable, or trainable, circuit, without increasing fabrication complexity. The neuromorphic inverter configuration (shown in Fig. 3c) allows for interactive tuning of the gain, a characteristic feature used to extract, for example, electrophysiological signals ${ }^{2}$. The conductance of the EC-RAM can be altered in a similar fashion as described before. The conductance states that are presented in Figure $3 \mathrm{~d}$ modulate the inverter behaviour and result in a shift of the inverter gain peak (see Fig. 3e).

Conventional complementary circuits based on a combination of a $p$ - and $n$-type material commonly require multiple or complex fabrication steps. Using our ambipolar conductive polymer we can significantly decrease this complexity by monolithic fabrication including the added neuromorphic traits. This expands the capabilities of the complementary circuits in bioelectronics and sensors, in which OMIECS have already proven to be powerful due to their large volumetric ionic capacitance that allows for a significant amplification when transducing biological signals. In biological, aqueous, environments stable p-type OMIECs are common, whereas high performance n-type materials, in terms of stability and electrical behaviour, are not. However, for biosensors detecting anions or some metabolites like glucose, stable n-type materials are crucial.

a)

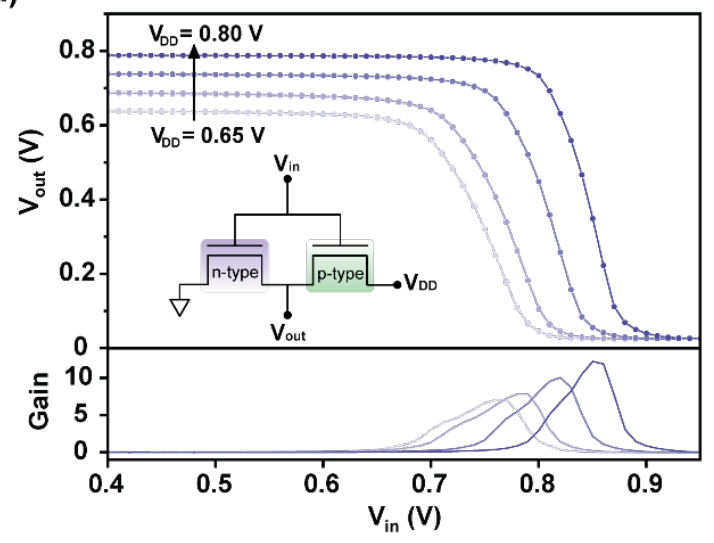

c)

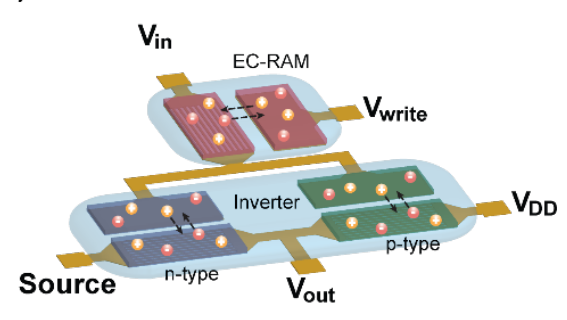

b)

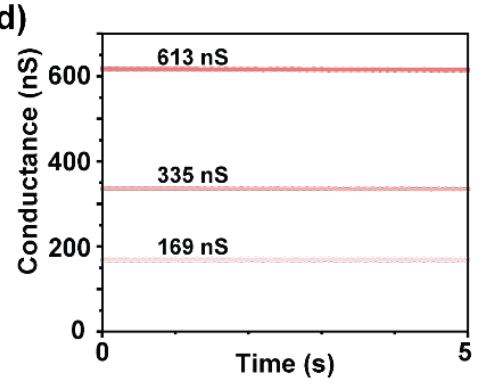

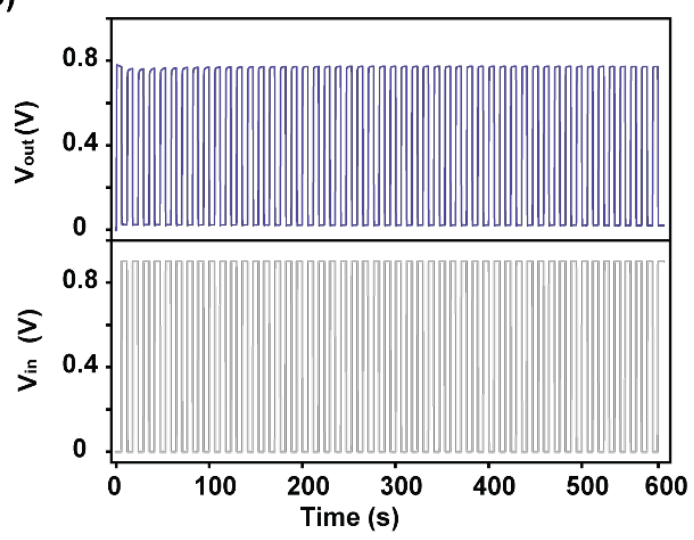

e)

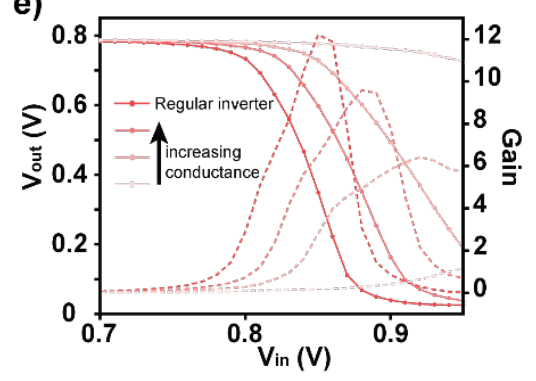

Figure 3| Solid-state ambipolar inverter. a) Ambipolar inverter behaviour at different $V_{D D}$ voltages with below the corresponding gain $\left(\left|\partial \mathrm{V}_{\text {out }} / \partial \mathrm{V}_{\text {in }}\right|\right)$ and the inset representing the 
schematic inverter circuit. b) Complementary logic gate behaviour of a NOT gate with $V_{D D}$ $=0.8 \mathrm{~V}$ and the input $V_{\text {in }}$ pulsing between 0 and $0.9 \mathrm{~V}$ for $6 \mathrm{~s}$. c) Illustration of a neuromorphic inverter including an EC-RAM in series with the gate of the ambipolar inverter. d) Read out of the different conductance states of the EC-RAM with e) the corresponding behaviour of the neuromorphic inverter $\left(\mathrm{V}_{\mathrm{DD}}=0.8 \mathrm{~V}\right)$.

We studied the behaviour of a $100 \mathrm{mM} \mathrm{NaCl}$ aqueous electrolyte gated OECT using P-3O as the active channel, and $\mathrm{Ag} / \mathrm{AgCl}$ as the gate electrode (shown in Fig. 4a). In this configuration P-3O also exhibits ambipolar behaviour in transistor performance (see Fig. 4b and 4c for the output and transfer curves), which indicates that the polymer could also be doped by both cations $\left(\mathrm{Na}^{+}\right)$and anions $\left(\mathrm{Cl}^{-}\right)$. In n-type mode, the $\mathrm{P}-30$ polymer works exceptionally well with stable on/off ratio of $\sim 4$ orders of magnitude for more than 21600 cycles, shown in Figure $4 \mathrm{~d}$. After continuously operating more than 60 hours, the on-current only exhibits a slight decrease (approximately 25\%) but maintains the on/off ratio in similar orders of magnitude (see Fig. S13). The remarkable stability is a result of the precise tailoring of the energy levels (LUMO $4.30 \mathrm{eV}$, see Fig. S10 and Table S1) with respect to oxygen and water ${ }^{19}$. Despite extremely high stability for p-type in ion-gel, the material performance in liquid electrolytes in p-type mode is less (see Fig. S14), showing 2 orders of magnitude on/off ratio for over 2 hours operation. The aqueous electrolyte operated ambipolar inverter and the tuning of it using the EC-RAM is demonstrated in Figure S22 and S24. We further show the exceptional n-type performance of the $\mathrm{P}-30$ polymer in aqueous electrolyte by demonstrating its behaviour as a neurotransmitter sensor (see Fig. 4e) which has potential in advanced biohybrid systems ${ }^{4}$. We adopted a $\mathrm{Au}$ side-gated device configuration in Figure $1 \mathrm{a}$ and monitored the drain current at a drain potential of $V_{d s}=0.1 \mathrm{~V}$, while oxidizing the dopamine at the gate electrode by applying a gate potential of $V_{g}=0.6 \mathrm{~V}$. A faradaic gate current was observed when different concentrations of dopamine analyte were added (Fig. 4e bottom panel), which lowers the potential drop at the Au gate and electrolyte interface, leading to more effective gating at the channel (See Fig. S15). Meanwhile, the drain current increases in response to increased dopamine concentrations (Fig. 4e top panel and Fig. S16, S17). These results prove the interaction of P-3O as an excellent electron conductor with neurotransmitters like dopamine, showing its potential in adaptive biohybrid applications.

a)

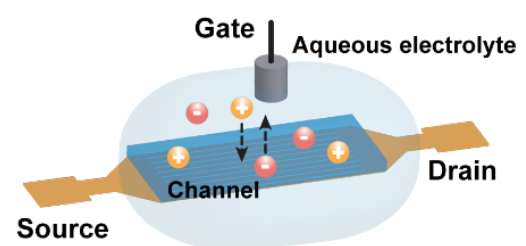

b)

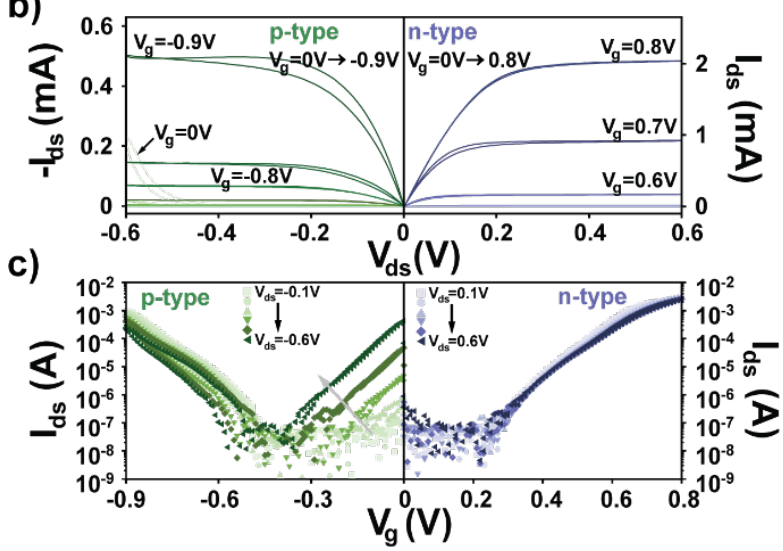

d)

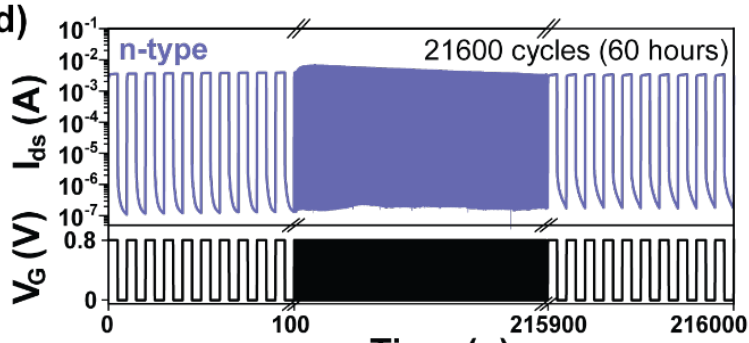

e)

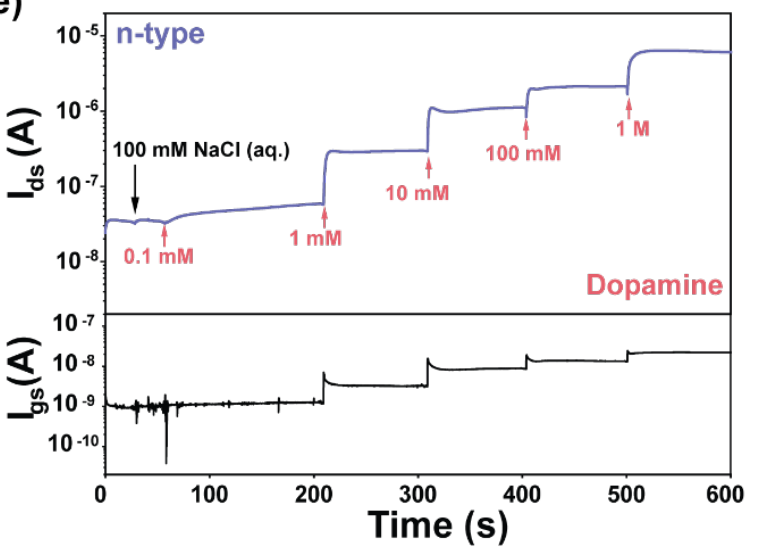


Figure 4| Aqueous operation of the P-30 based devices. a) Schematic of aqueous electrolyte gated OECT. b) Ambipolar P-3O OECT output curve. c) Ambipolar P-3O OECT semi-log transfer curve. d) N-type OECTs $\left(V_{g}=0.8 \mathrm{~V}, V_{d s}=0.5 \mathrm{~V}\right)$ operation stability in $100 \mathrm{mM}$ $\mathrm{NaCl}$ aqueous electrolyte for 21600 pulse cycles. A gate voltage pulse $V_{g}$ was applied for $5 \mathrm{~s}$ with an interval time of $5 \mathrm{~s}$. e) Au side-gated OECTs for dopamine sensing. The output drain current $I_{\mathrm{ds}}$, gate current $I_{\text {gs }}$ were monitored when adding different concentration of dopamine into the $100 \mathrm{mM} \mathrm{NaCl}$ (aq.) electrolyte at $\mathrm{V}_{\mathrm{ds}}=0.1 \mathrm{~V}$ and $\mathrm{V}_{\mathrm{g}}=0.6 \mathrm{~V}$. A glass well was glued to the device using PDMS to retain the electrolyte. Starting with $40 \mu \mathrm{L} 100 \mathrm{mM} \mathrm{NaCl}$ aqueous solution. $10 \mu \mathrm{L}$ analyte solution was added into the well each time.

Due to the intrinsic ionic-electronic coupling, OMIECs exhibit great potential not only in bridging electronics and biological systems, but also in constructing non-volatile memory devices for hardware artificial neural networks (ANNs). In this work, we have presented the capability to integrate individual devices into circuits for potential applications like adaptive biosensors and brain-inspired computing, utilizing the ambipolar NDI-thiazole conjugated polymer P-30. The remarkable characteristics of this semiconducting polymer, including symmetric $\mathrm{p}$ - and $\mathrm{n}$-type behaviour, high performance and ambient stability, allows for straightforward monolithic fabrication and integration of single devices, including transistors, neuromorphic memory and sensors, as well as logic gates and trainable circuits for nextgeneration adaptive and personalized bioelectronic applications.

\section{Experimental}

Materials. The P-30 synthesis details and characterization are described in the Supplementary Information. Chloroform, 1-ethyl-3-methylimidazolium bis(trifluorosulfonyl)imide, poly(vinylidene fluoride-co-hexafluoropropylene), and dopamine hydrochloride are purchased from Sigma-Aldrich and used as received. The ion gel solution was prepared following the reported method ${ }^{24}$ without further baking the ionic liquid. Ionic liquid (EMIM:TFSI) and poly(vinylidene fluoride-co-hexafluoropropylene) $(4: 1 \mathrm{w} / \mathrm{w})$ were dissolved in acetone with the following proportions: $17.6 \mathrm{wt} \%$ ionic liquid, $4.4 \mathrm{wt} \%$ polymer and $78 \mathrm{wt} \%$ solvent. The resulting ion gel solution was stirred at $40{ }^{\circ} \mathrm{C}$ for at least $30 \mathrm{~min}$. The interdigitated microelectrodes IDA-Au-6 (channel length $\mathbf{L} 5 \mu \mathrm{m}$, individual channel width w $1.8 \mathrm{~mm}$, number of pairs 30 , number of channels $\mathbf{n} 59$, total channels width $\mathbf{W}=n \cdot w=10.62 \mathrm{~cm}$ ) are purchased from MicruX technologies.

OECTs, EC-RAMs Fabrication and Characterization. The polymer solutions were prepared in chloroform at the concentration of $5 \mathrm{mg} / \mathrm{mL}$. The interdigitated microelectrodes were treated with UV ozone over 15 mins, following spin-coating the polymer solutions at $1000 \mathrm{rpm}$ for $30 \mathrm{~s}$. The samples were annealed on the hotplate at $100{ }^{\circ} \mathrm{C}$ for 30 mins. For side gate devices, the gate and active channel were separated by excimer laser ablation. The ion gel solution was drop casted on the top of the active area and dried in the fume hood. For EC-RAM measurements, the devices were stored in the nitrogen atmosphere glovebox. The electrical characterization of OECTs is recorded by a Keithley sourcemeter SMU 2602B, which is controlled by the software Arkeo developed by Cici research. The EC-RAMs are recorded by Keithley 2602B with Labview and the inverter data is acquired with a NI DAQ USB-6363 and with Keithley SMU 2636B controlled with Matlab software.

\section{Data availability}

The data are available from the authors on reasonable request.

\section{Acknowledgements}

Y.Z, E.R.W.D and Y.v.d.B. gratefully acknowledges funding from the European Union's Horizon 2020 Research and Innovation Programme, grant agreement no. 802615. G.Y. and J.S. acknowledge the National Natural Science Foundation of China (61620106016/61835009/61775145). G. Y. also acknowledges the China Postdoctoral 
Science Foundation Funded Project (grant 2020M672771) and Guangdong Basic and Applied Basic Research Foundation (2020A1515110636).

\section{Author contributions}

Y.Z., G.Y., J.S., and Y.v.d.B initiated the idea. Y.v.d.B. supervised the project. G.Y., J.S., and R.C.C. designed the polymer. G.Y. synthesized and characterized the polymer. Y.Z, E.R.W.D. and Y.v.d.B. designed the experiments. Y.Z, E.R.W.D performed device fabrications and the measurements. T.S. performed the CrossSim simulation. Y.Z., E.R.W.D. and G.Y. prepared the manuscript. Y.Z., E.R.W.D and Y.v.d.B. wrote the manuscript. All the authors contributed to the comments and suggestions on the final manuscript.

\section{Competing financial interests}

The authors declare no competing financial interests.

\section{References}

1. Koklu, A. et al. Organic Bioelectronic Devices for Metabolite Sensing. Chem. Rev. (2021) doi:10.1021/acs.chemrev.1c00395.

2. Cea, C. et al. Enhancement-mode ion-based transistor as a comprehensive interface and real-time processing unit for in vivo electrophysiology. Nat. Mater. 19, 679-686 (2020).

3. van de Burgt, Y. et al. A non-volatile organic electrochemical device as a low-voltage artificial synapse for neuromorphic computing. Nat. Mater. 16, 414-418 (2017).

4. Keene, S. T. et al. A biohybrid synapse with neurotransmitter-mediated plasticity. Nat. Mater. 19, 969-973 (2020).

5. Maria, P. A. et al. Direct metabolite detection with an n-type accumulation mode organic electrochemical transistor. Sci. Adv. 4, eaat0911 (2021).

6. Sun, H. et al. Complementary Logic Circuits Based on High-Performance n-Type Organic Electrochemical Transistors. Adv. Mater. 30, 1704916 (2018).

7. Khodagholy, D. et al. In vivo recordings of brain activity using organic transistors. Nat. Commun. 4, 1575 (2013).

8. Khodagholy, D. et al. High transconductance organic electrochemical transistors. Nat. Commun. 4, 2133 (2013).

9. Romele, P. et al. Multiscale real time and high sensitivity ion detection with complementary organic electrochemical transistors amplifier. Nat. Commun. 11, 3743 (2020).

10. He, R.-X. et al. Detection of bacteria with organic electrochemical transistors. J. Mater. Chem. 22, 22072-22076 (2012).

11. Guo, K. et al. Rapid single-molecule detection of COVID-19 and MERS antigens via nanobody-functionalized organic electrochemical transistors. Nat. Biomed. Eng. 5, 666-677 (2021).

12. Gkoupidenis, P., Schaefer, N., Garlan, B. \& Malliaras, G. G. Neuromorphic Functions in PEDOT:PSS Organic Electrochemical Transistors. Adv. Mater. 27, 7176-7180 (2015).

13. Keene, S. T., Melianas, A., van de Burgt, Y. \& Salleo, A. Mechanisms for Enhanced State Retention and Stability in Redox-Gated Organic Neuromorphic Devices. Adv. Electron. Mater. 5, 1800686 (2019).

14. Gkoupidenis, P., Koutsouras, D. A. \& Malliaras, G. G. Neuromorphic device architectures with global connectivity through electrolyte gating. Nat. Commun. 8, 15448 (2017).

15. Fuller, E. J. et al. Parallel programming of an ionic floating-gate memory array for scalable neuromorphic computing. Science (80-. ). 364, 570 LP - 574 (2019).

16. Nielsen, C. B. et al. Molecular Design of Semiconducting Polymers for HighPerformance Organic Electrochemical Transistors. J. Am. Chem. Soc. 138, 1025210259 (2016).

17. Keene, S. T. et al. Enhancement-Mode PEDOT:PSS Organic Electrochemical 
Transistors Using Molecular De-Doping. Adv. Mater. 32, 2000270 (2020).

18. Ohayon, D. et al. Biofuel powered glucose detection in bodily fluids with an n-type conjugated polymer. Nat. Mater. 19, 456-463 (2020).

19. Griggs, S., Marks, A., Bristow, H. \& McCulloch, I. n-Type organic semiconducting polymers: stability limitations, design considerations and applications. J. Mater. Chem. C 9, 8099-8128 (2021).

20. Surgailis, J. et al. Mixed Conduction in an N-Type Organic Semiconductor in the Absence of Hydrophilic Side-Chains. Adv. Funct. Mater. 31, 2010165 (2021).

21. Liu, J. et al. N-Type Organic Thermoelectrics of Donor-Acceptor Copolymers: Improved Power Factor by Molecular Tailoring of the Density of States. Adv. Mater. 30, (2018).

22. Wang, S. et al. A Chemically Doped Naphthalenediimide-Bithiazole Polymer for $\mathrm{n}-$ Type Organic Thermoelectrics. Adv. Mater. 30, 1-6 (2018).

23. Liu, Q. et al. Fully Printed All-Solid-State Organic Flexible Artificial Synapse for Neuromorphic Computing. ACS Appl. Mater. Interfaces 11, 16749-16757 (2019).

24. Lee, K. H. et al. "Cut and Stick" Rubbery Ion Gels as High Capacitance Gate Dielectrics. Adv. Mater. 24, 4457-4462 (2012).

25. Melianas, A. et al. Temperature-resilient solid-state organic artificial synapses for neuromorphic computing. Sci. Adv. 6, eabb2958 (2020).

26. Choi, Y., Oh, S., Qian, C., Park, J.-H. \& Cho, J. H. Vertical organic synapse expandable to 3D crossbar array. Nat. Commun. 11, 4595 (2020).

27. Quill, T. J. et al. Ion Pair Uptake in Ion Gel Devices Based on Organic Mixed IonicElectronic Conductors. Adv. Funct. Mater. n/a, 2104301 (2021).

28. Cox, J. A. et al. A Signal Processing Approach for Cyber Data Classification with Deep Neural Networks. Procedia Comput. Sci. 61, 349-354 (2015).

29. Sekitani, T. et al. Ultraflexible organic amplifier with biocompatible gel electrodes. Nat. Commun. 7, 11425 (2016).

30. Chen, J. et al. Printed subthreshold organic transistors operating at high gain and ultralow power. Science (80-. ). 363, 719-723 (2019).

31. Rashid, R. B. et al. Ambipolar inverters based on cofacial vertical organic electrochemical transistor pairs for biosignal amplification. Sci. Adv. 7, eabh1055 (2021). 


\section{Table of Contents}

\section{Supplementary Information}

1. Synthetic Procedures

2. Organic Electrochemical Transistors (OECTs)

3. Electrochemical Resistive Random-access Memory devices EC-RAMs

4. Ambipolar inverters

5. EC-RAM based neural network simulation

\section{Synthetic Procedures}

\section{1-1 Synthesis and characterization of materials}

\section{Reagents}

All reagents and solvents were commercial and were used as received. 2,6-dibromo-1,4,5,8naphthalene tetracarboxylic diimide was purchased from TCl. 4,4'-bis(octyloxy)-2,2'bis(trimethylstannyl)-5,5'-bithiazole (2Tz) was purchased from SunaTech. 2-(2-(2mthoxyethoxy)ethoxy)ethanamine and bis(2-(2-(2ethoxyethoxy)ethoxy)ethyl)benzo[Imn][3,8]phenanthroline-1,3,6,8(2H,7H)-tetraone (NDI-30) were synthesized according to literature procedures ${ }^{\mathrm{S1}-\mathrm{S} 2}$.

\section{Characterization}

${ }^{1} \mathrm{HNMR}$ and ${ }^{13} \mathrm{CNMR}$ were performed on a Varian Unity Plus $(400 \mathrm{MHz})$ instrument at $25^{\circ} \mathrm{C}$, using tetramethylsilane (TMS) as an internal standard. NMR shifts are reported in ppm, relative to the residual protonated solvent signals of $\mathrm{CDCl}_{3}(\delta=7.26 \mathrm{ppm})$ or at the carbon absorption in $\mathrm{CDCl}_{3}(\delta=77.23 \mathrm{ppm})$. Multiplicities are denoted as: singlet (s), doublet (d), triplet $(\mathrm{t})$ and multiplet $(\mathrm{m})$. High Resolution Mass Spectroscopy (HRMS) was performed on a JEOL JMS 600 spectrometer. FT-IR spectra were recorded on a Nicolet Nexus FT-IR fitted with a Thermo Scientific Smart iTR sampler. Thermal properties of the polymers were determined on a TA Instruments DSC Q20 and a TGA Q50. DSC measurements were executed with two heating-cooling cycles with a scan rate of $10^{\circ} \mathrm{C} \mathrm{min}{ }^{-1}$. TGA measurements were done from 20 to $800{ }^{\circ} \mathrm{C}$ with a heating rate of $20^{\circ} \mathrm{C} \mathrm{min}{ }^{-1}$. Cyclic voltammetry (CV) was carried out with a $\mathrm{CH}$-Instruments $760 \mathrm{e}$ Electrochemical Workstation in a three-electrode configuration where the working electrode was glassy carbon electrode, the counter electrode was a platinum wire, and the pseudo-reference was a $\mathrm{Ag} / \mathrm{Ag}^{+}$reference electrode that was calibrated against ferrocene $\left(\mathrm{Fc} / \mathrm{Fc}^{+}\right)$. Cyclic voltammograms for the polymer films deposited on the glassy carbon working electrode in $\mathrm{CHCN}_{3}$ solution containing $\mathrm{Bu}_{4} \mathrm{NPF}_{6}\left(0.1 \mathrm{~mol} \mathrm{~L}^{-1}\right)$ electrolyte at a scanning rate of $100 \mathrm{mV} \mathrm{s}^{-1}$.<smiles>O=C1OC(=O)c2c(Br)cc3c4c(c(Br)cc(c24)C1=O)C(=O)OC3=O</smiles>
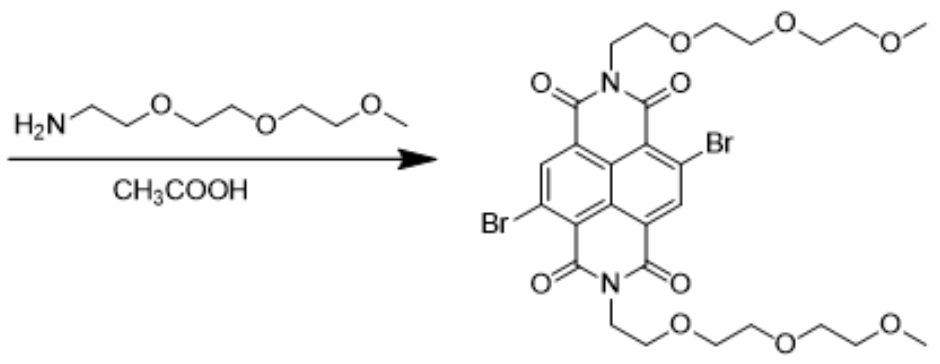

Figure S1: Synthetic route for NDI based monomer NDI-30. 


\section{Synthesis of NDI-30}

2-(2-(2-methoxyethoxy)ethoxy)ethanamine $(600 \mathrm{mg}, 3.38 \mathrm{mmol}, 4 \mathrm{eq})$ was added to a suspension of 2,6-dibromo-1,4,5,8-naphthalene tetracarboxylic diimide (360 mg, $0.845 \mathrm{mmol}$, $1 \mathrm{eq}$ ) in $10 \mathrm{~mL}$ of glacial acetic acid and heated to $120^{\circ} \mathrm{C}$ for $4 \mathrm{~h}$. The reaction mixture was then cooled to room temperature and added to water, then extracted with $\mathrm{CH}_{2} \mathrm{Cl}_{2}$. The organic phase was washed with brine, dried over anhydrous sodium sulfate and removed solvent by rotary evaporator. The crude product was purified by silica gel column chromatography with $\mathrm{CH}_{2} \mathrm{Cl}_{2}$ /acetone as the eluent afforded target compound as a bright yellow solid.

NDI-30: ${ }^{1} \mathrm{HNMR}\left(400 \mathrm{MHz}, \mathrm{CDCl}_{3}\right)$ : $\delta 8.97(\mathrm{~s}, 2 \mathrm{H}), 4.46(\mathrm{t}, \mathrm{J}=5.6 \mathrm{~Hz}, 4 \mathrm{H}), 3.84$ (t, J= $6.0 \mathrm{~Hz}$ $4 \mathrm{H}), 3.73-3.67(\mathrm{~m}, 4 \mathrm{H}), 3.65-3.52(\mathrm{~m}, 8 \mathrm{H}), 3.52-3.42(\mathrm{~m}, 4 \mathrm{H}), 3.31(\mathrm{~s}, 6 \mathrm{H}) .{ }^{13} \mathrm{CNMR}(100 \mathrm{MHz}$, $\left.\mathrm{CDCl}_{3}\right): \delta 163.47,163.37,141.66,130.97,130.40,127.96,126.74,74.51,73.25,73.16,72.74$, 70.22, 61.64, 42.67. HRMS(ESI) Calcd. for $\mathrm{C}_{28} \mathrm{H}_{32} \mathrm{Br}_{2} \mathrm{~N}_{2} \mathrm{O}_{10} \mathrm{Na}_{1}[\mathrm{M}+\mathrm{Na}]^{+}:$739.02954, found: 739.03025 .

a)

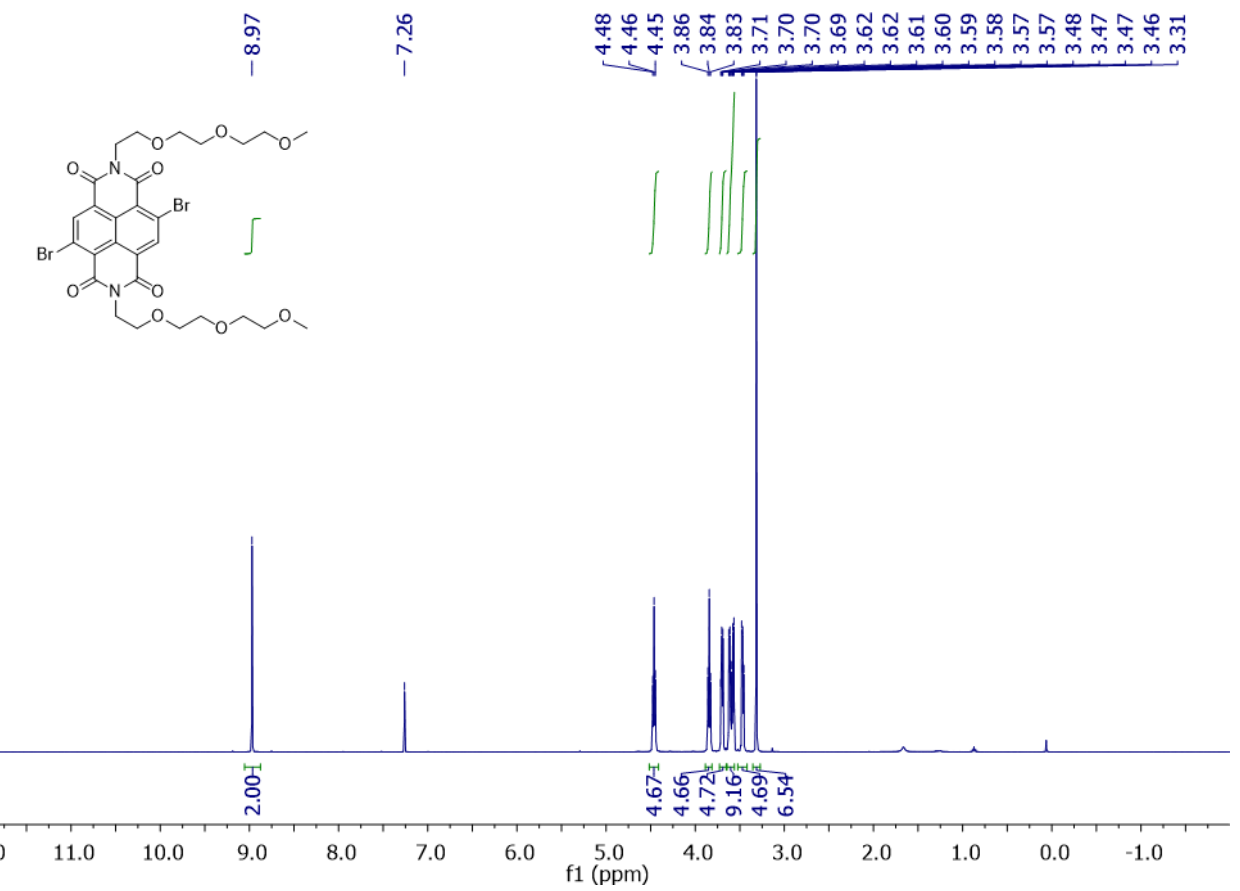


b)

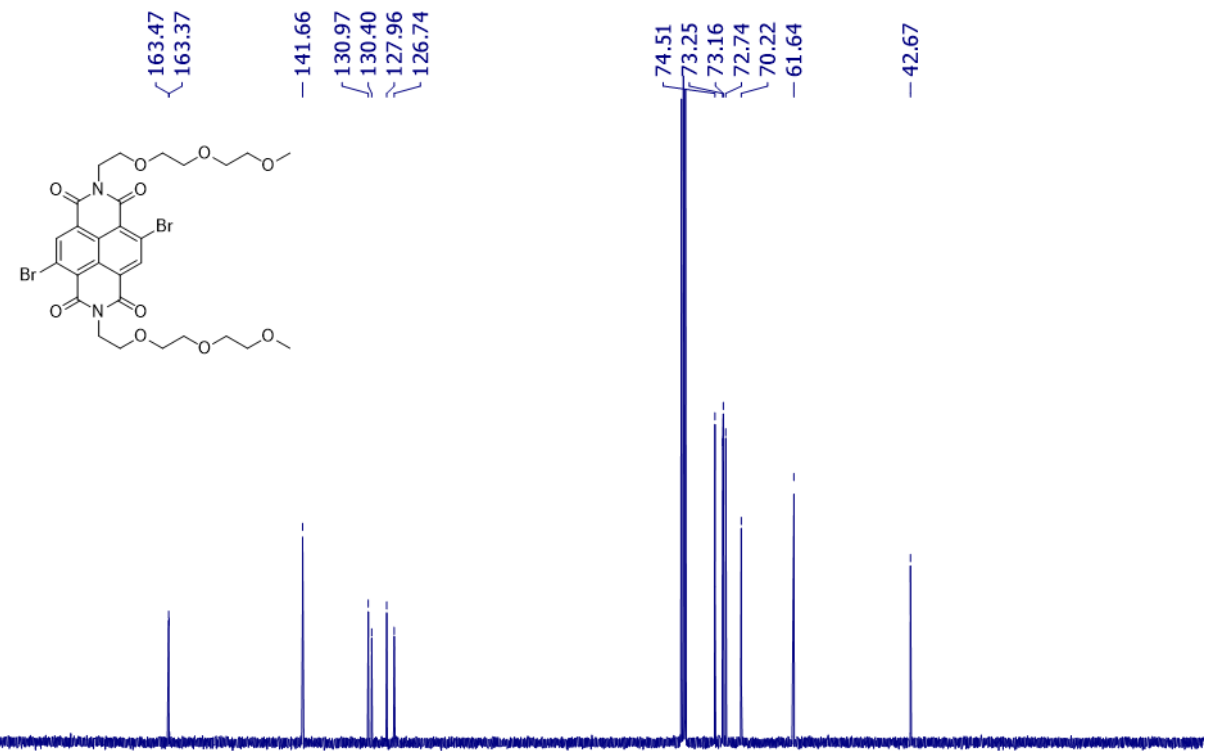

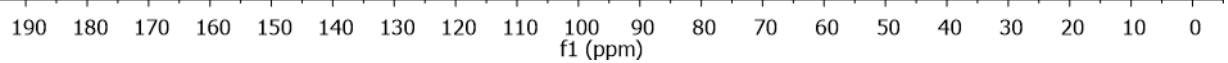

Figure S2: a) ${ }^{1} \mathrm{HNMR}$ and b) ${ }^{13} \mathrm{CNMR}$ spectra of NDI-30

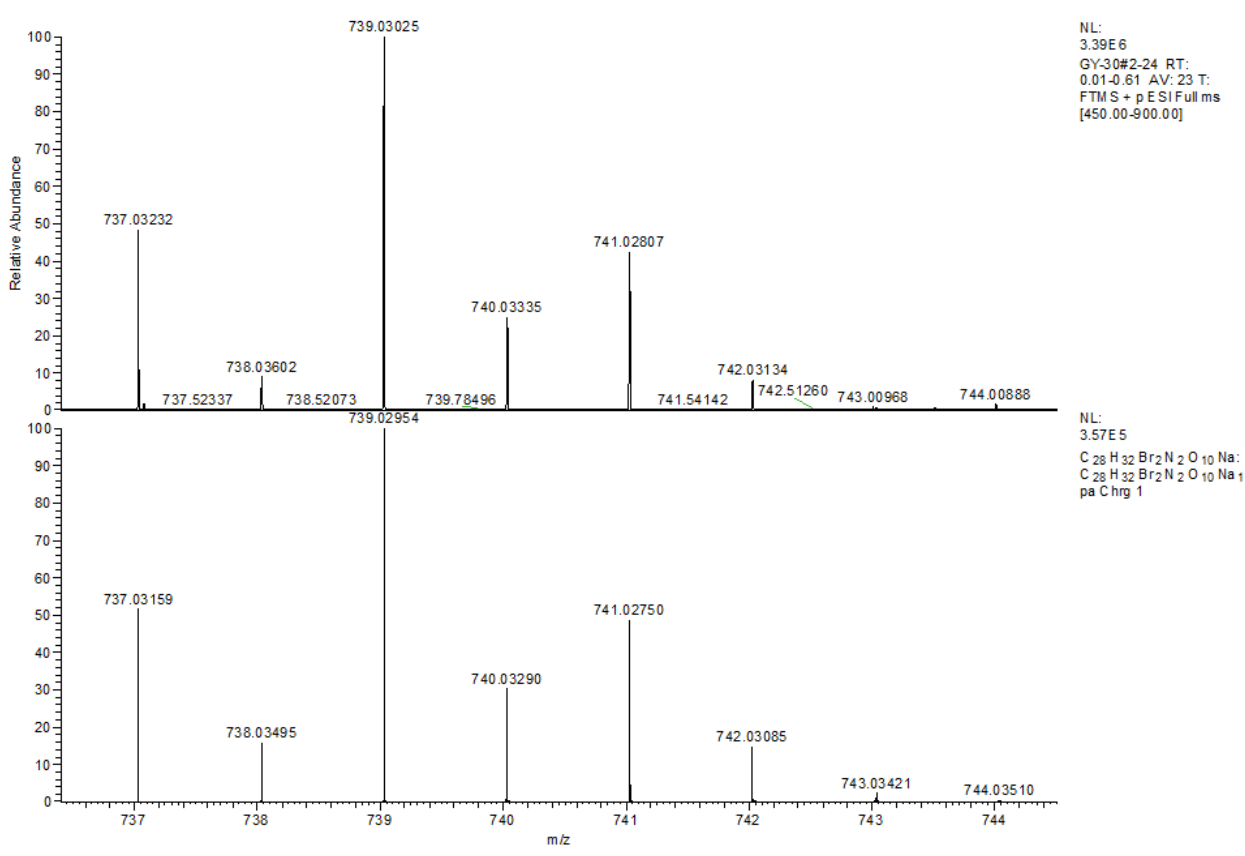

Figure S3: HRMS spectra of NDI-30

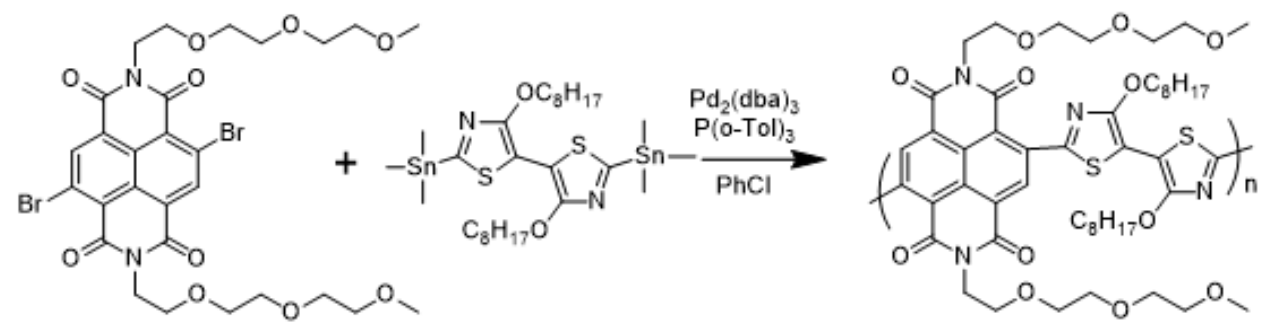


Figure S4: Synthetic route for NDI-Tz based low band gap conjugated polymer P-30.

\section{Synthetic procedures for the NDI-Tz (PNDI-30) based polymers}

To a dry three-neck flask, NDI based monomer NDI-30 $(0.1 \mathrm{mmol}, 71.6 \mathrm{mg})$ and thiazole based monomer $2 \mathrm{Tz}(0.1 \mathrm{mmol}, 75 \mathrm{mg})$ were added under argon followed by tris(dibenzylideneacetone) dipalladium $\left[\mathrm{Pd}_{2}(\mathrm{dba})_{3}\right](8 \mathrm{mg})$ and tri(o-tolyl)phosphine $\left[\mathrm{P}(\mathrm{o} \text {-tolyl })_{3}\right]$ (12 mg). The flask and its contents were subjected to 3 pump/purge cycles with $\mathrm{N}_{2}$ followed by addition of anhydrous, degassed chlorobenzene $(5 \mathrm{~mL})$ via syringe. The reaction mixture was stirred at $120^{\circ} \mathrm{C}$ for five days. After cooling to room temperature, the deeply gray coloured reaction mixture was dropped into $100 \mathrm{~mL}$ vigorously stirred methanol (containing $5 \mathrm{~mL} 12 \mathrm{M}$ hydrochloride acid). After stirring for 4 hours, the precipitated solid was collected by filtration. The solid polymers were redissolved in chloroform and reprecipitated into methanol. After filtration, the polymers were subjected to sequential Soxhlet extraction. The sequential solvents were methanol, hexane and chloroform. Impurities and low-molecular-weight fractions were removed by methanol and hexane. Finally, the polymer solution in chloroform was concentrated to give the polymers as dark solid. ${ }^{1} \mathrm{HNMR}\left(400 \mathrm{MHz}, \mathrm{CDCl}_{3}\right)$ : $\delta 10.38-8.79$ (m, 2H), 5.08-2.66 (m, 34H), 2.12-0.62 (m, 30H). IR ( $\left.\mathrm{cm}^{-1}\right): 668,692,719,763,790,896,950$, $982,1064,1104,1176,1208,1245,1297,1314,1366,1441,1487,1564,1647,1698,2854$, 2920.

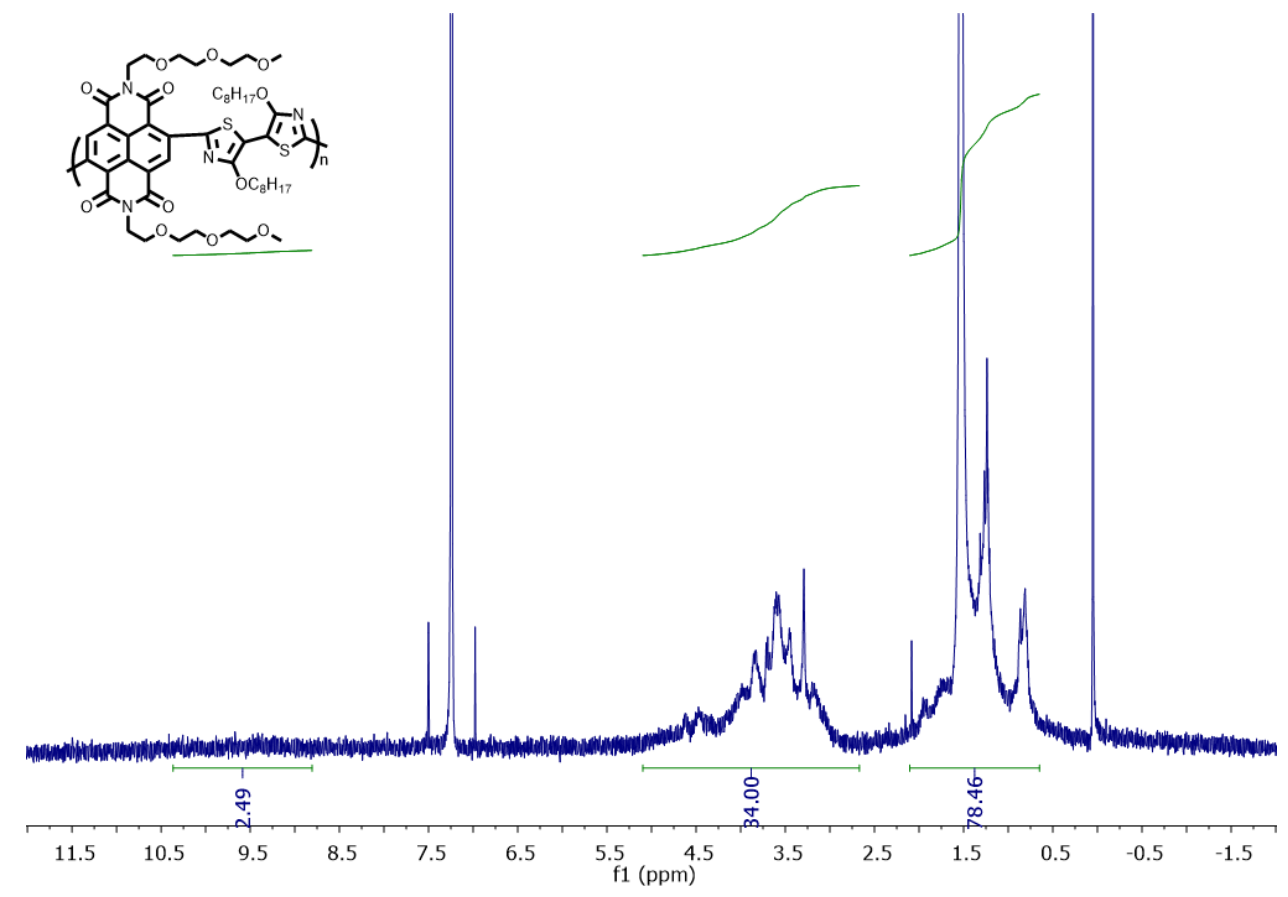

Figure S5: ${ }^{1} \mathrm{HNMR}$ spectra of NDI based polymer (P-3O). 


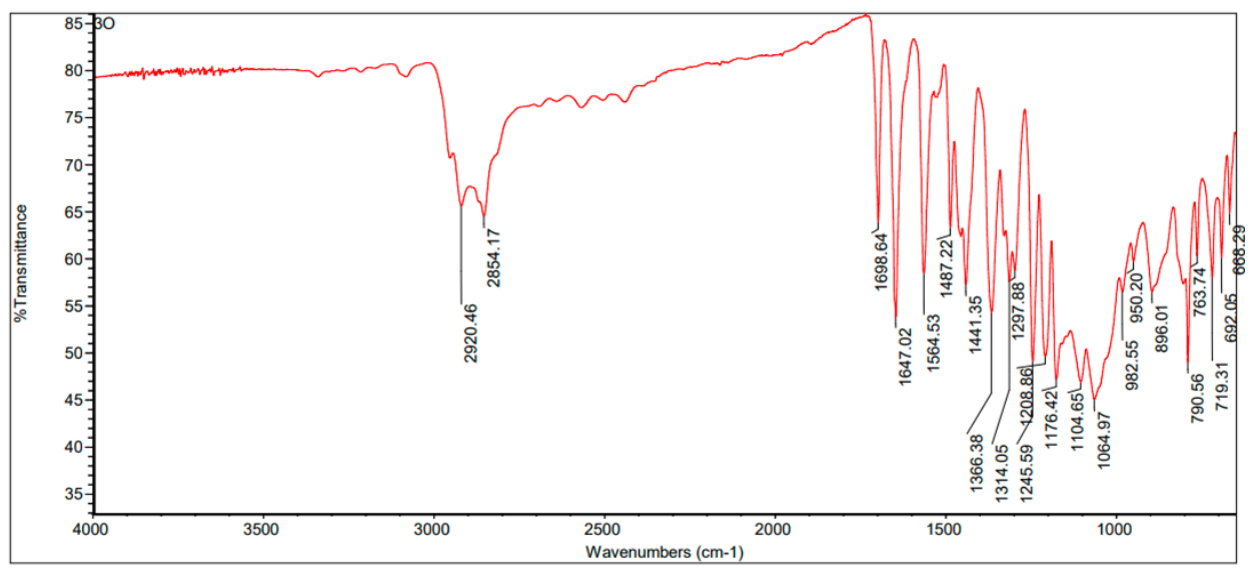

Figure S6: IR spectra of NDI based polymer (P-3O).

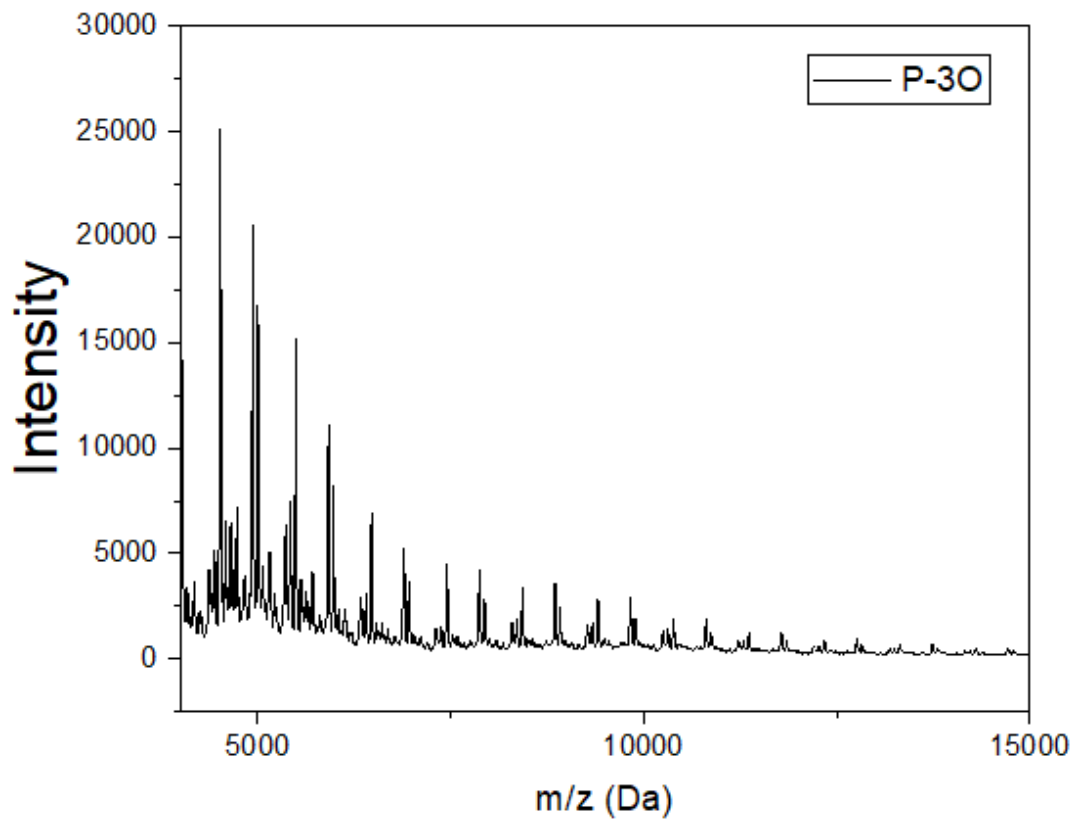




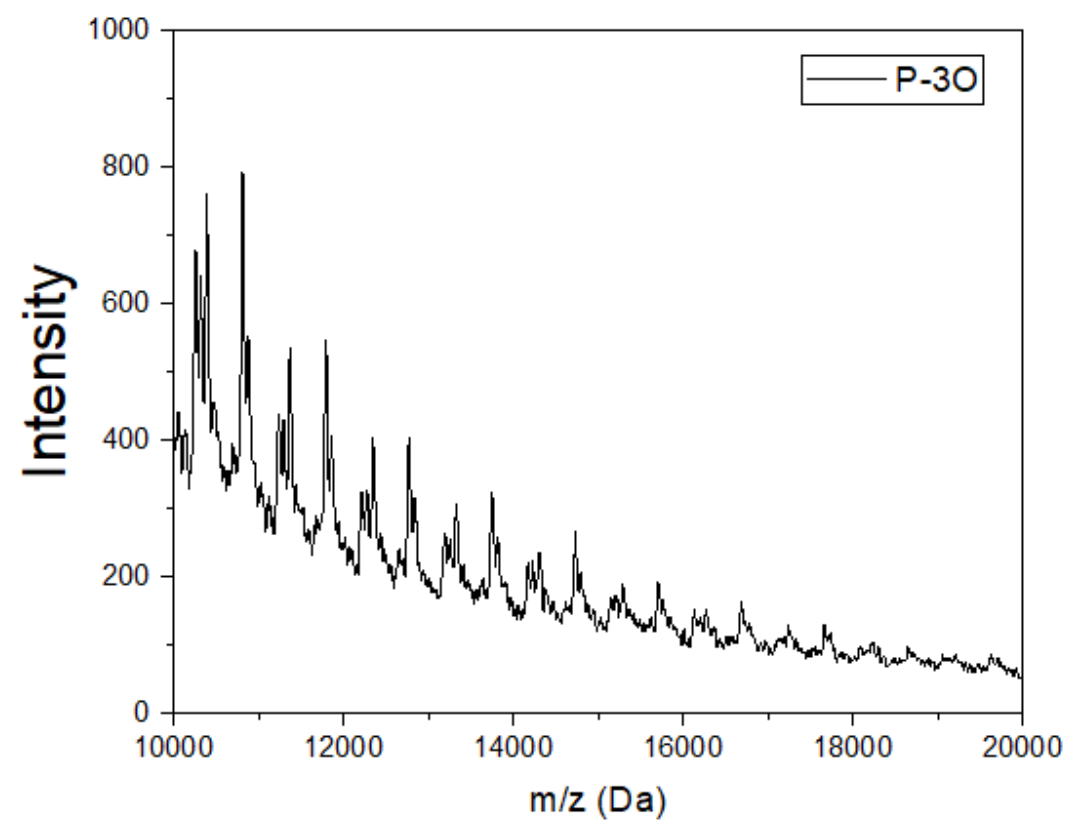

Figure S7. MALDI-TOF-MS spectra of NDI based polymer P-3O.

\section{1-2 Thermal properties}

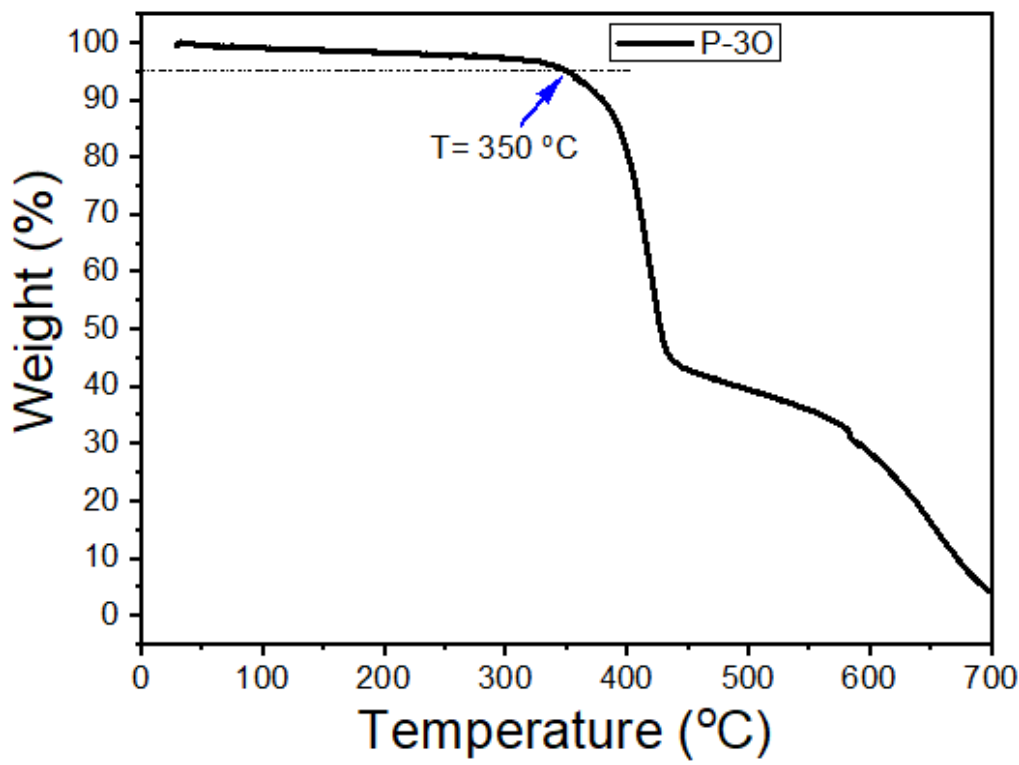




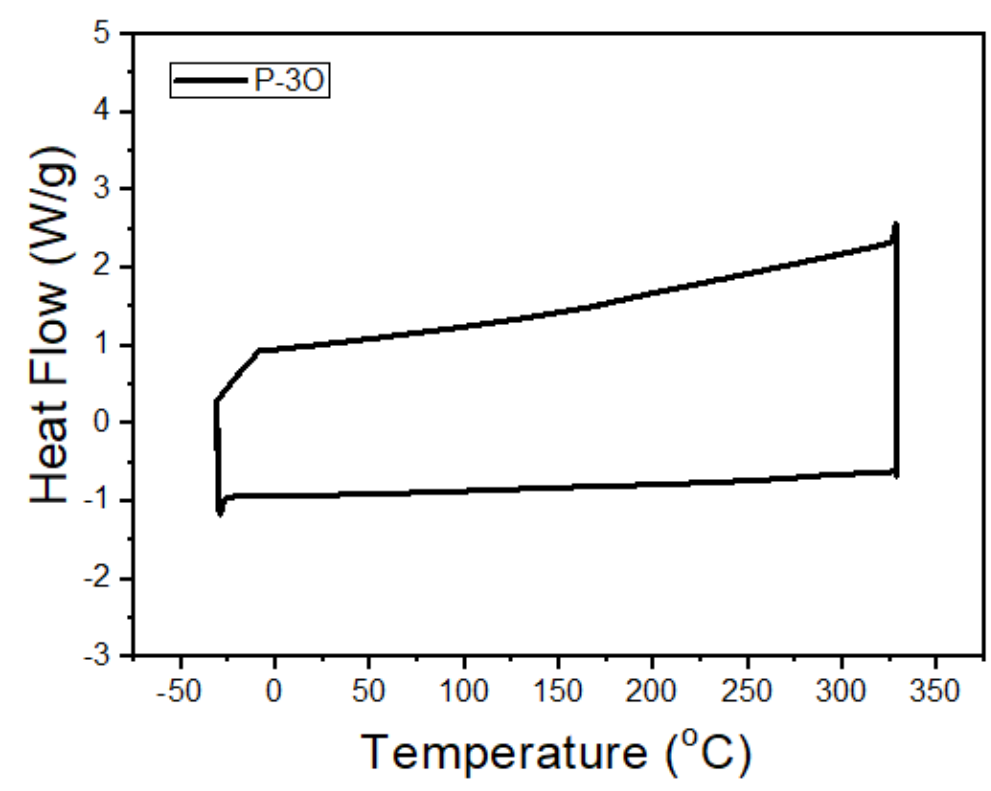

Figure S8: Thermogravimetric (TGA) analysis plots and DSC analysis plots for NDI based polymers (P-3O).

\section{1-3 Absorption properties}

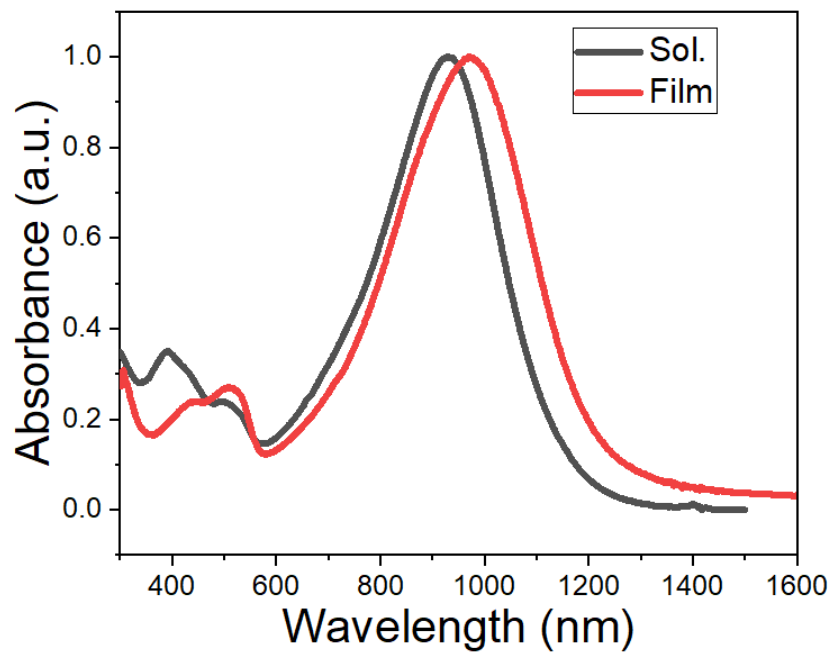

Figure S9: The absorption of NDI based polymers (P-3O) in dilute $\mathrm{CHCl}_{3}\left(10^{-5} \mathrm{M}\right)$ and thin film state.

\section{1-4 Electronchemistry properties}



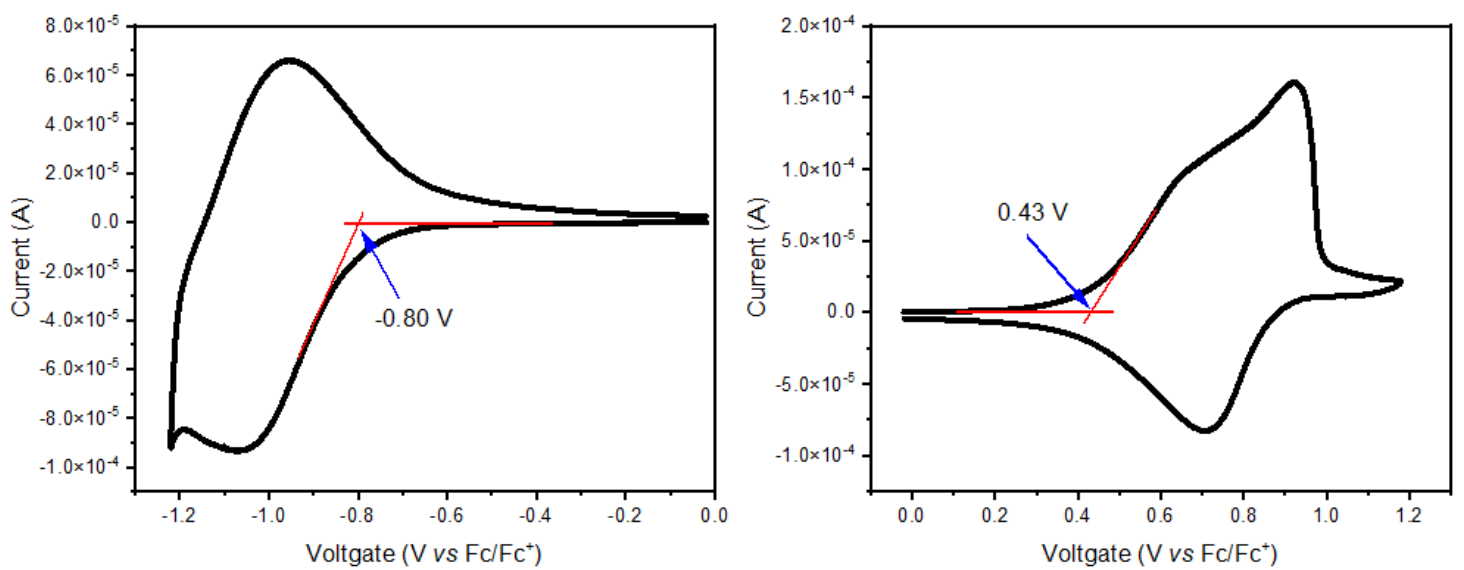

Figure S10. Cyclic voltammograms of the conjugated polymer P-30 thin films deposited on the glass carbon working electrode in $\mathrm{CHCN}_{3}$ solution containing $\mathrm{Bu}_{4} \mathrm{NPF}_{6}$ electrolyte.

Table S1. Optical and electrochemical data for the polymer P-3O.

\begin{tabular}{|c|c|c|c|c|c|c|c|c|}
\hline Polymer & $\begin{array}{c}\lambda_{\max } \text { film }[ \\
n m]\end{array}$ & $\begin{array}{c}\lambda_{\text {onset }^{\text {film }}} \\
{[\mathrm{nm}]}\end{array}$ & $\begin{array}{c}\mathrm{E}_{\mathrm{g}}^{\text {opt. (a) }} \\
{[\mathrm{eV}]}\end{array}$ & $\begin{array}{l}\text { HOMO } \\
\text { (b) }[\mathrm{eV}]\end{array}$ & $\begin{array}{l}\text { LUMO } \\
\text { (c) }[\mathrm{eV}]\end{array}$ & $\begin{array}{c}E_{\text {onset }^{\text {ox. }}[} \\
V]\end{array}$ & $\begin{array}{c}\mathrm{E}_{\text {onset }}^{\text {red. }}[ \\
\mathrm{V}]\end{array}$ & $\begin{array}{l}E_{g}{ }^{e c} \\
{[e V]}\end{array}$ \\
\hline $\mathrm{P}-30$ & 973 & 1225 & 1.01 & -5.53 & -4.30 & 0.43 & -0.80 & 1.23 \\
\hline
\end{tabular}

(a) $\mathrm{E}_{g}{ }^{\text {opt. }}=1240 / \lambda_{\text {onset }}$ film

(b) $\mathrm{HOMO}=-\left(5.10+E_{\text {onset }}{ }^{\text {red. }}\right) \mathrm{eV}$

(c) $L U M O=-\left(5.10+E_{\text {onset }}{ }^{\text {ox. }}\right) \mathrm{eV}$

\section{OECTS}

\section{2-1 lon gel gated OECTs}

a)

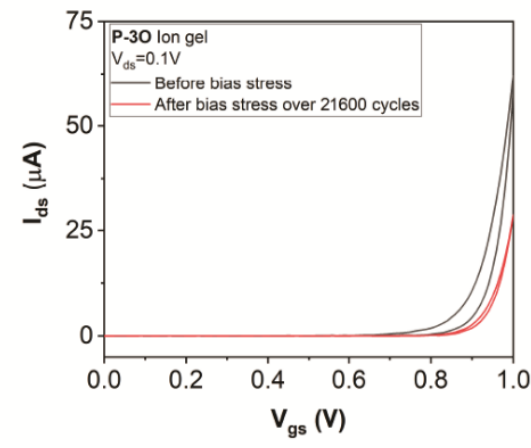

b)

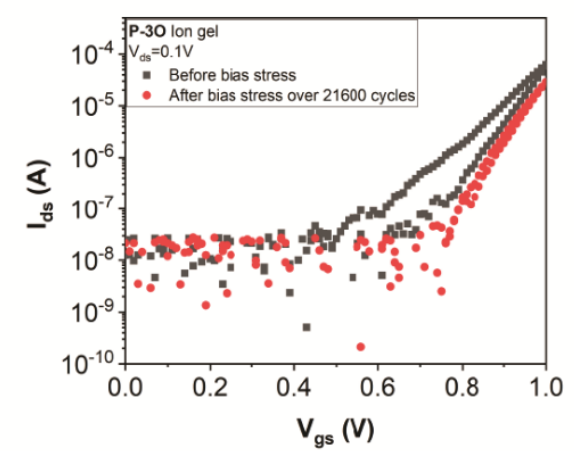

Figure S11. Comparison of P-3O ion gel OECT n-type transfer curve $\left(\mathrm{V}_{\mathrm{ds}}=0.1 \mathrm{~V}\right)$ before and after bias stress stability over 21600 cycles (pulse 5s, interval 5s, over 60 hours). a) Linear plot, b) Semi-log plot. 
a)

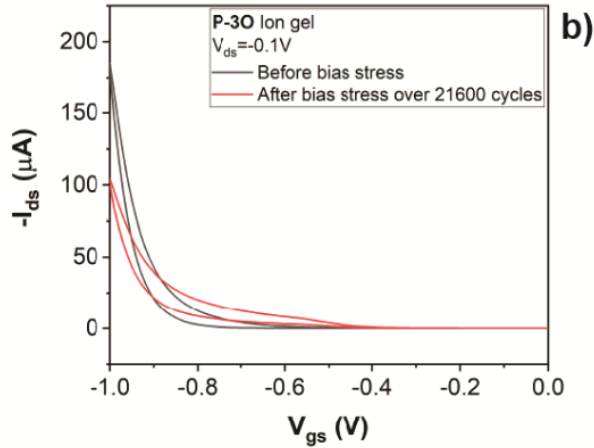

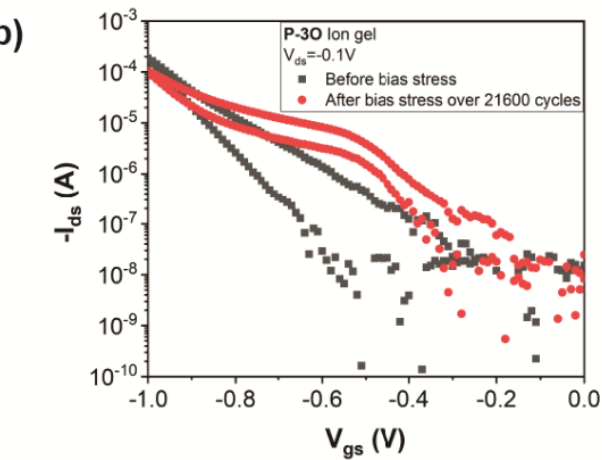

Figure S12. Comparison of P-3O ion gel OECT p-type transfer curve $\left(\mathrm{V}_{\mathrm{ds}}=-0.1 \mathrm{~V}\right)$ before and after bias stress stability over 21600 cycles (pulse 5s, interval 5s, over 60 hours). a) Linear plot, b) Semi-log plot.

\section{2-2 Aqueous electrolyte OECT}

a)

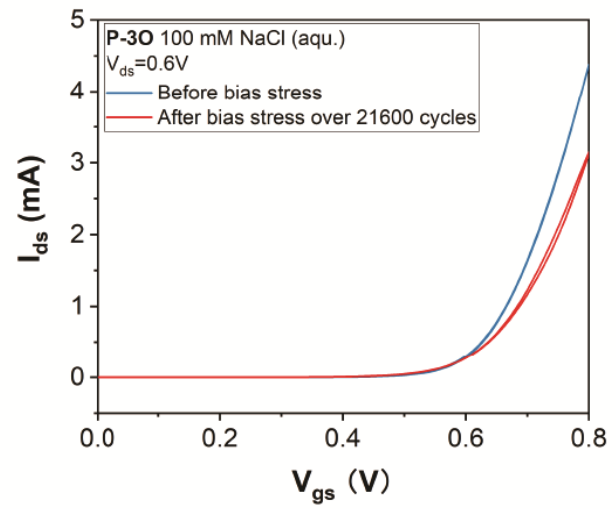

b)

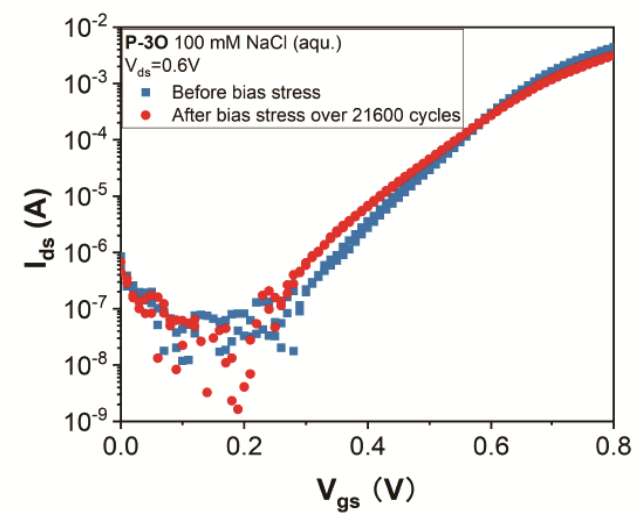

Figure S13. Comparison of P-30 $100 \mathrm{mM} \mathrm{NaCl}$ aqueous OECT transfer curve $\left(\mathrm{V}_{\mathrm{ds}}=0.6 \mathrm{~V}\right)$ before and after bias stress stability over 21600 cycles (pulse $5 \mathrm{~s}$, interval 5s, over 60 hours). a) Linear plot, b) Semi-log plot.

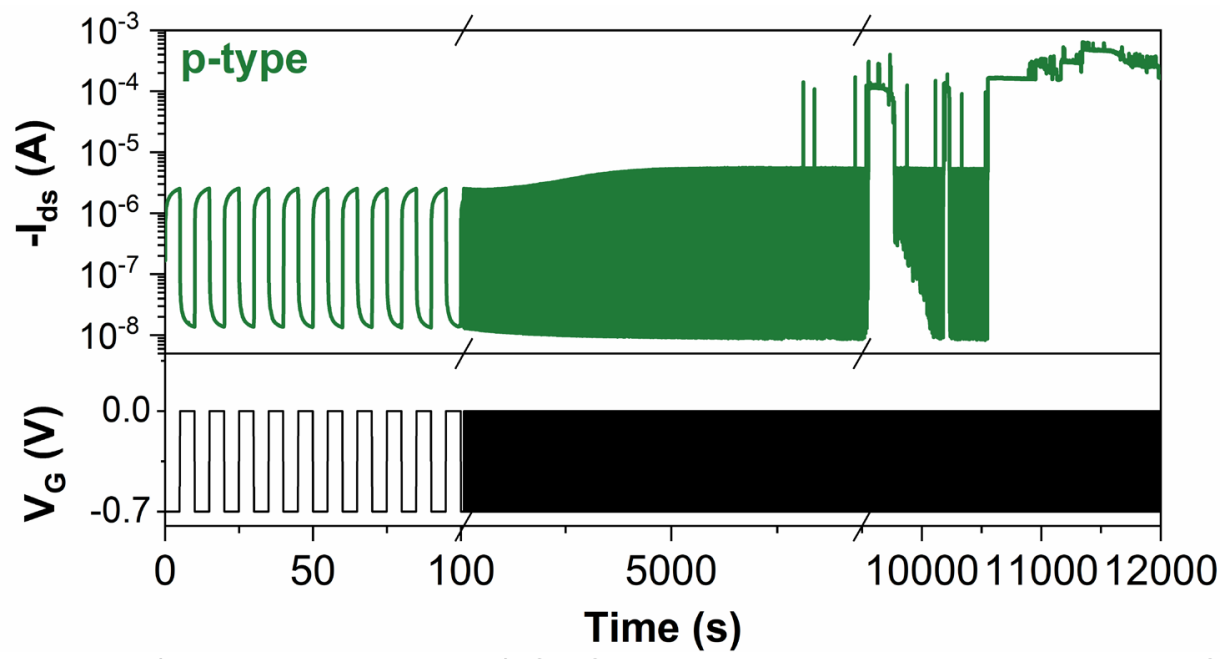

Figure $\mathrm{S} 14$. P-type $\left(\mathrm{V}_{\mathrm{g}}=-0.7 \mathrm{~V}, \mathrm{~V}_{\mathrm{ds}}=-0.1 \mathrm{~V}\right) \mathrm{OECTs}$ operation stability in $100 \mathrm{mM} \mathrm{NaCl}$ aqueous electrolyte. A gate voltage pulse $\mathrm{V}_{\mathrm{g}}$ was applied for $5 \mathrm{~s}$ with an interval time of $5 \mathrm{~s}$. The device became instable after 950 cycles and broke down eventually. 


\section{2-3 Dopamine sensing}
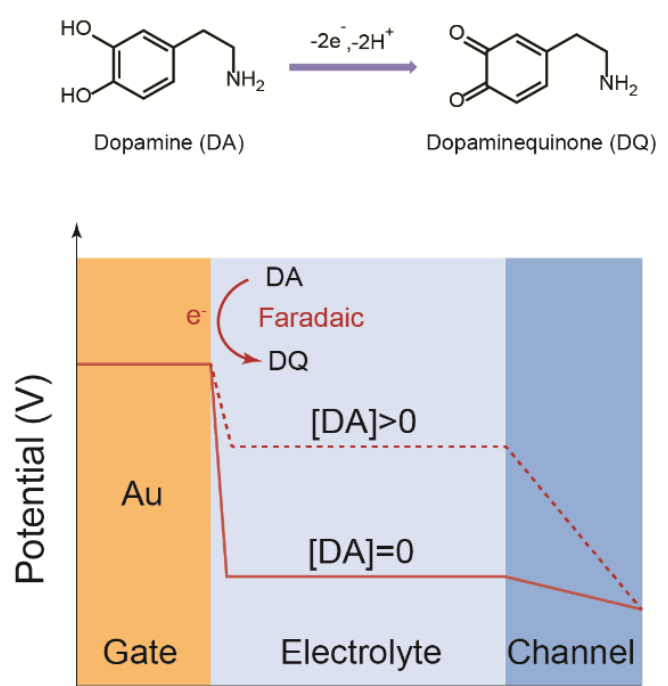

Figure S15. Dopamine sensing mechanism. Dopamine (DA) can be electrochemical oxidized to its quinone form (DQ). This schematic illustrates the potential drop across the $A u$ gate OECTs system. When a gate potential (higher than the oxidation potential of DA) is applied, Dopamine is oxidized electrochemically at the Au gate electrode. The oxidation of DA generates Faradaic currents, which lower the potential drop across the Au/electrolyte interface, leading to more effective gating at the active channel.

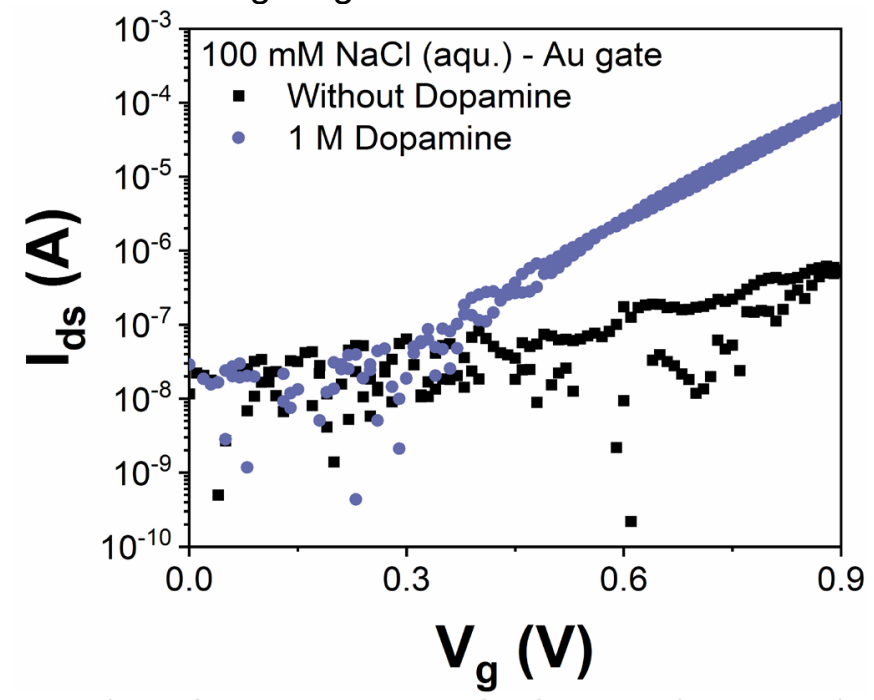

Figure S16. Comparison of $\mathrm{P}-3 \mathrm{O}$ Au side-gated OECT transfer curve $\left(\mathrm{V}_{\mathrm{ds}}=0.1 \mathrm{~V}\right)$ with and without $1 \mathrm{M}$ dopamine in $100 \mathrm{mM} \mathrm{NaCl}$ aqueous electrolyte. 
a) $1 \mathrm{M}$ Dopamine in $100 \mathrm{mM} \mathrm{NaCl}$ (aqu.) - Au gate

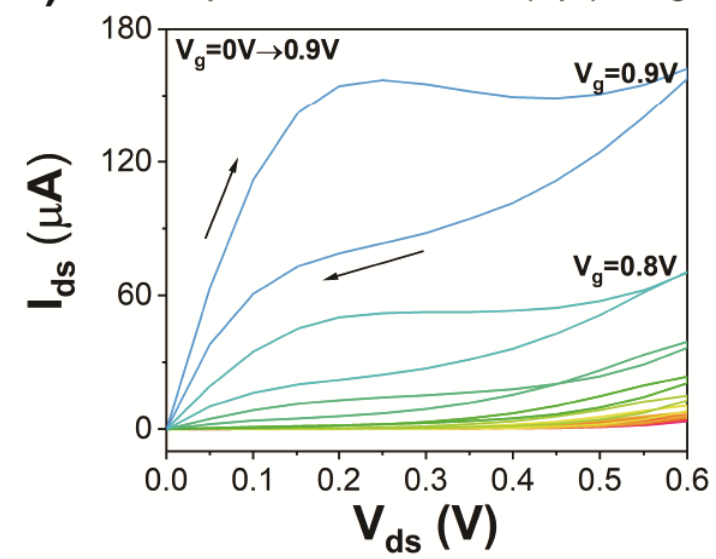

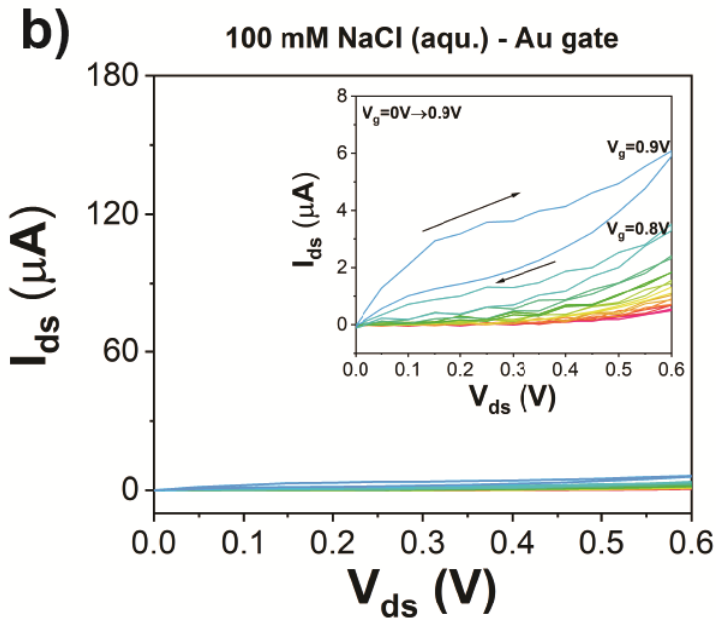

Figure S17. Comparison of P-3O Au side-gated OECT output curve a) with $1 \mathrm{M}$ dopamine, b) without dopamine in $100 \mathrm{mM} \mathrm{NaCl}$ aqueous electrolyte.

\section{EC-RAMs}

a)

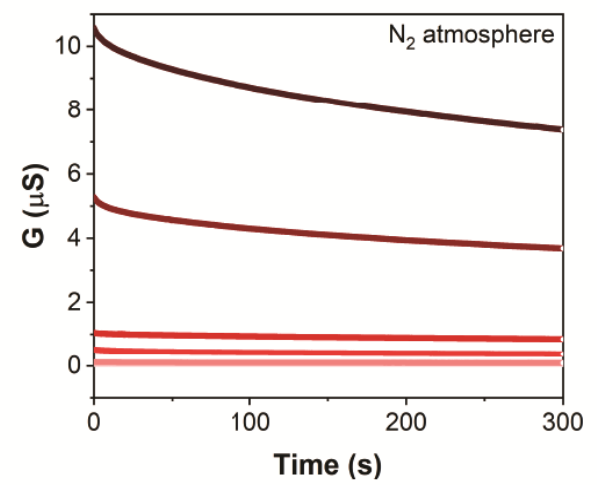

b)

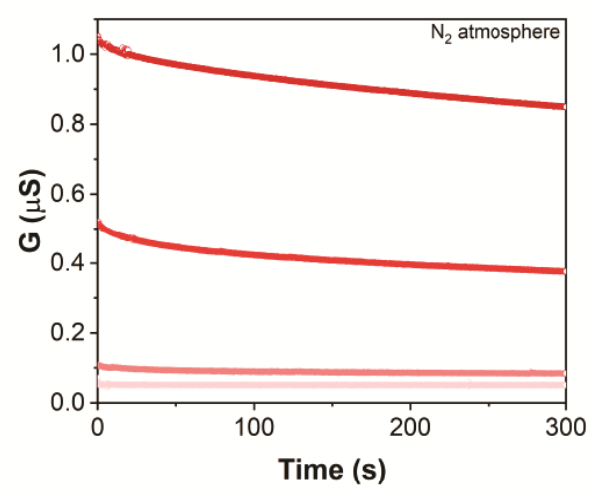

Figure S18. Linear plot of P-30 EC-RAMs n-type conductance state retention over time (5 mins).

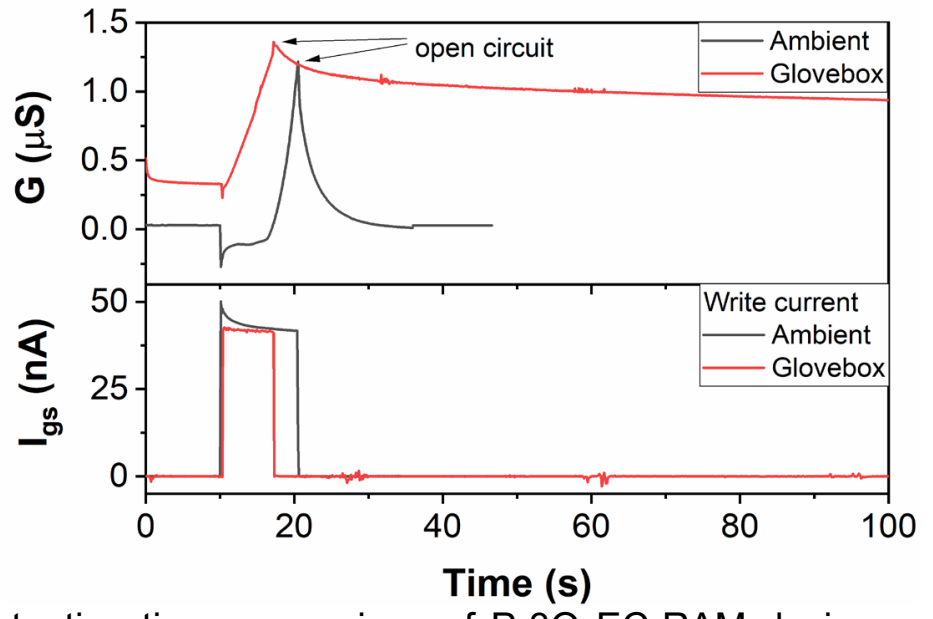

Figure S19. The retention time comparison of P-3O EC-RAM devices $\mathbf{n}$-type operation in ambient and $\mathrm{N}_{2}$ atmosphere glovebox. The conductance state decays faster when oxygen and water present $\left(\mathrm{V}_{\mathrm{ds}}=0.1 \mathrm{~V}\right)$. 


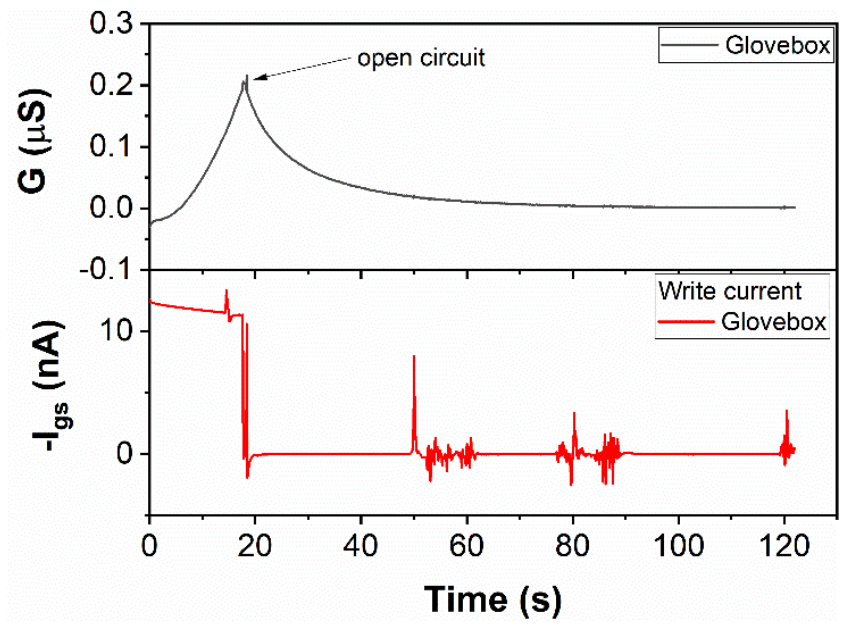

Figure S20. The retention time of P-30 EC-RAM devices p-type operation in $\mathrm{N}_{2}$ atmosphere glovebox. The conductance state decays very fast $\left(V_{d s}=-0.1 \mathrm{~V}\right)$.

\section{Ambipolar inverter}

4.1

The ambipolar inverter is realized by connecting two single ion-gated transistors with a shared ion gel or aqueous electrolyte. One of the two channels of the Keithley SMU 2636B is used to supply the input voltage $\left(V_{\text {in }}\right)$ whereas the other channel is used for the potential at $V_{D D}$. Each of these voltages including the output voltage $\left(\mathrm{V}_{\text {out }}\right)$ is recorded with a DAQ NI USB-6353. The operation with either a solid ion gel or aqueous electrolyte shows the transition from a high to low output at a different input value. This correlates to the different values of the threshold voltage obtained from the transfer curve (Figure S11 and S13). The higher gain for aqueous electrolyte gated inverters can be explained by the higher on/off ratio for the individual OECT devices. Due to the increased conductance at the gate-electrolyte interface the voltage drop across this interface is reduced and the gating at the channel is more effective compared to the ion-gel gated equivalent.

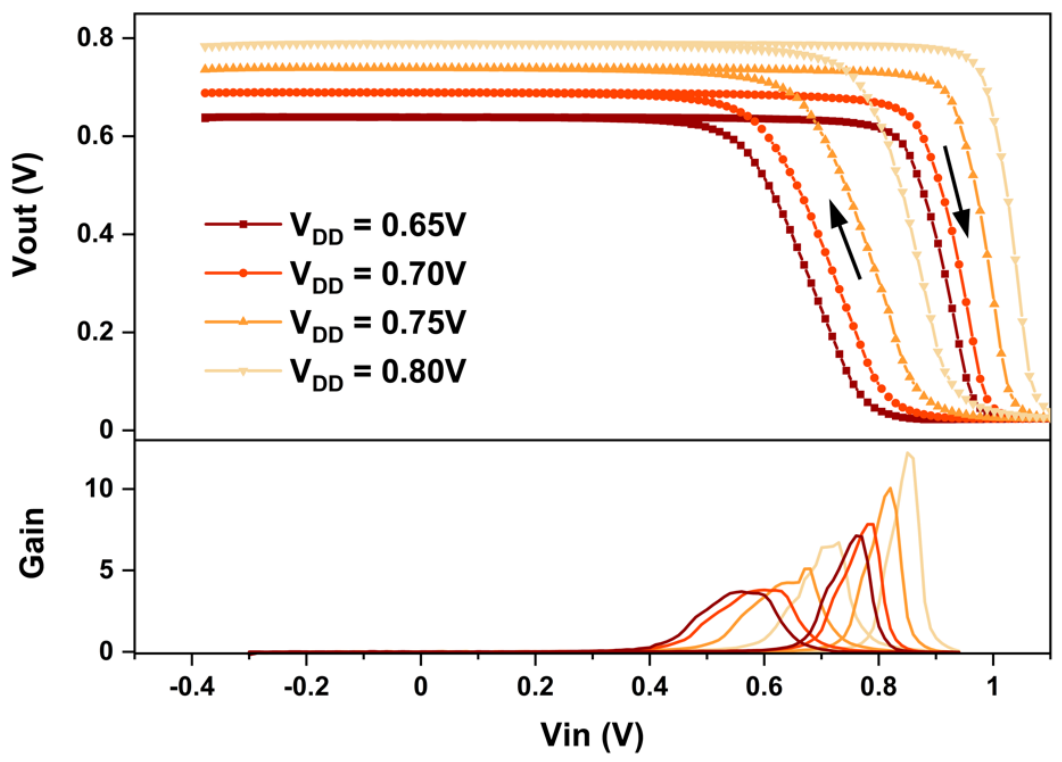

Figure S21. Solid state ambipolar inverter behavior for increasing $\mathrm{V}_{\mathrm{DD}}(0.65 \mathrm{~V}$ to $0.80 \mathrm{~V})$. Input voltage $\mathrm{V}_{\text {in }}$ is scanned from $-0.3 \mathrm{~V}$ to $1.1 \mathrm{~V}$ and back $(0.01 \mathrm{~V} / \mathrm{s})$. 


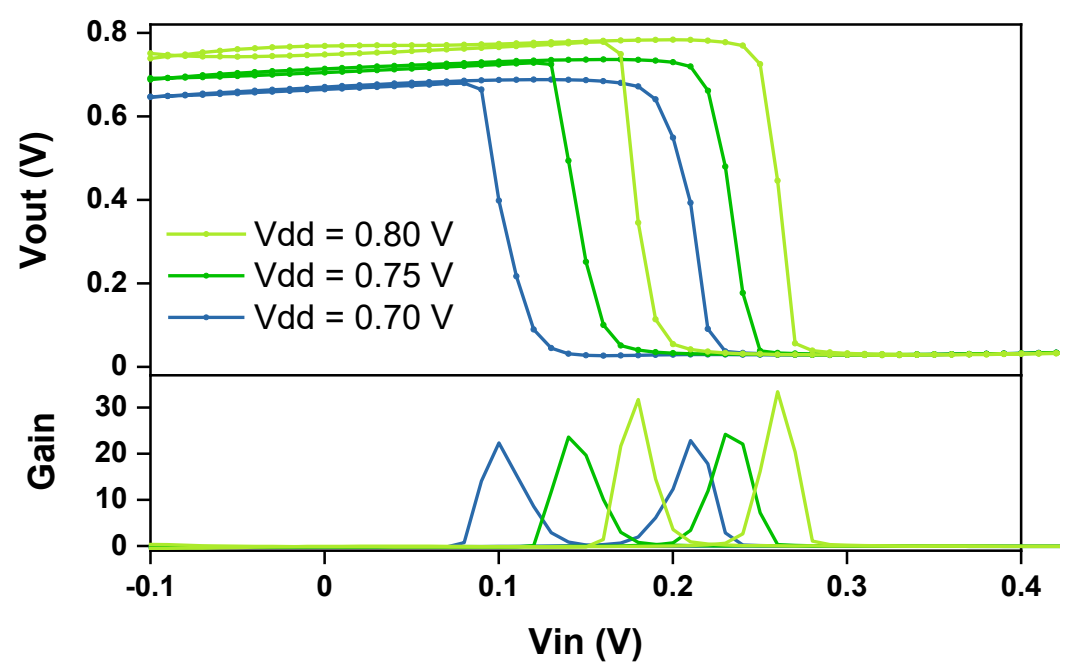

Figure S22. Aqueous gated ambipolar inverter behavior for increasing $\mathrm{V}_{\mathrm{DD}}(0.70 \mathrm{~V}$ to $0.80 \mathrm{~V})$. Input voltage $\mathrm{V}_{\text {in }}$ is scanned from $-0.2 \mathrm{~V}$ to $0.5 \mathrm{~V}$ and back $(0.01 \mathrm{~V} / \mathrm{s})$.

4.2

The neuromorphic ambipolar inverter is configured in a similar fashion as the regular ambipolar inverter described in SI.4.1, with the difference that the EC-RAM channel is in series with the gate (previously $V_{\text {in }}$ ) of the regular inverter. The conductance state of the EC-RAM is modified with Keithley SMU 2602B using LabView software.

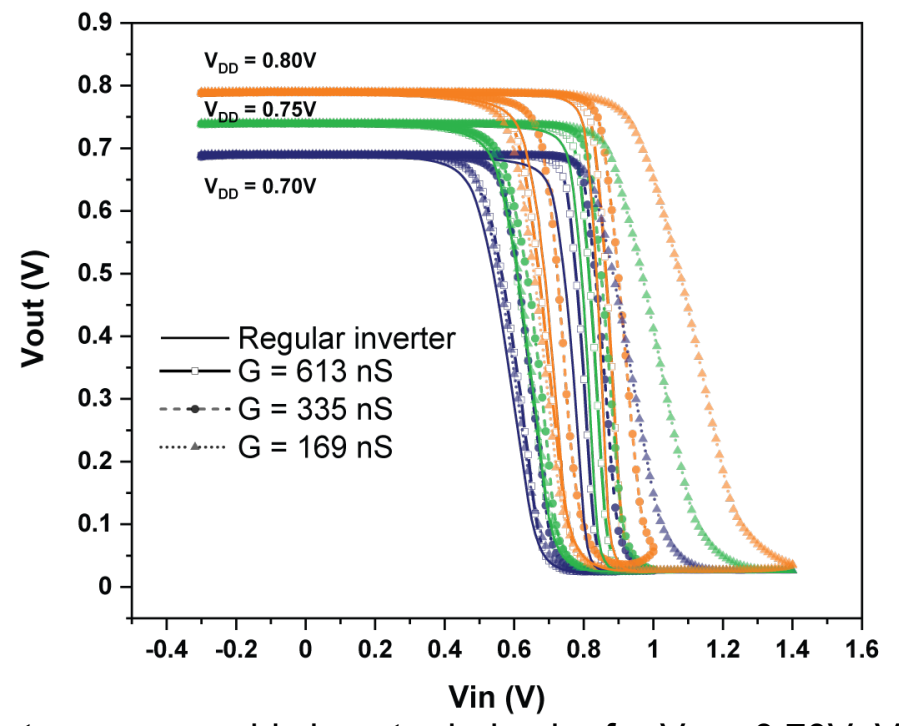

Figure S23. Solid-state neuromorphic inverter behavior for $V_{D D}=0.70 \mathrm{~V}, V_{D D}=0.75 \mathrm{~V}$, and $V_{D D}$ $=0.80 \mathrm{~V}$ with each scan a decreasing EC-RAM conductance. Input voltage $V_{\text {in }}$ is scanned from $-0.3 \mathrm{~V}$ to $1.0 \mathrm{~V}$ and back $(0.01 \mathrm{~V} / \mathrm{s})$. For the sweep at the lowest conductance value $\mathrm{V}_{\text {in }}$ is scanned to $1.4 \mathrm{~V}$ in order to completely turn the inverter output off. 


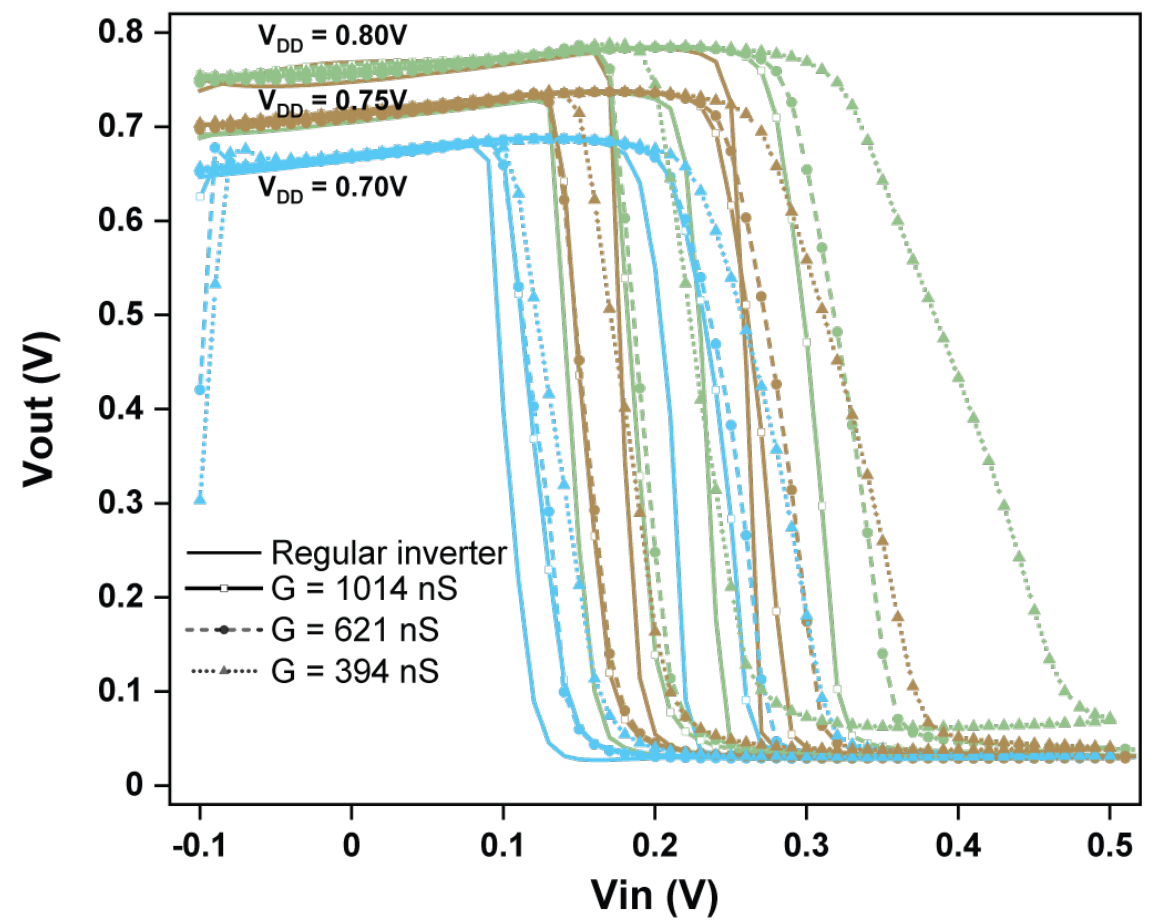

Figure S24. Aqueous neuromorphic inverter behavior for $V_{D D}=0.70 \mathrm{~V}, V_{D D}=0.75 \mathrm{~V}$, and $V_{D D}$ $=0.80 \mathrm{~V}$ with each scan a decreasing EC-RAM conductance. Input voltage $V_{\text {in }}$ is scanned from $-0.1 \mathrm{~V}$ to $0.5 \mathrm{~V}$ and back $(0.01 \mathrm{~V} / \mathrm{s})$.

\section{EC-RAM based neural network simulation}

In order to verify the EC-RAM's ability to function as the weight in a neural network, the classification performance of crossbar array of these devices is examined. A model of a crossbar array is simulated using CrossSim (Sandia National laboratories) ${ }^{\mathrm{S} 5}$ with the same

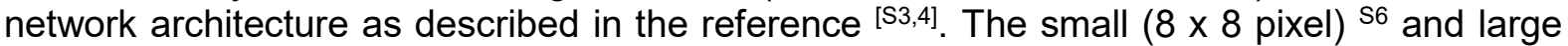
$\left(28 \times 28\right.$ pixel) ${ }^{\text {S7 }}$ MNIST handwritten digits datasets are used and classified with a $64 \times 36 \times$ 10 and $784 \times 300 \times 10$ network structure, respectively.

The experimental weights are extracted from the conductance modulation measurement (Figure 2d) with 14 potentiation and depression cycles of 32 measured states. In the simulation the weights are offset by $50 \%$ to introduce negative weight values and the total range is clipped by $25 \%$ (maximum and minimum) to enable operation in the most linear regime. Both datasets are trained for 40 epochs with a sigmoid activation function. To validate the efficacy of the ECRAM based crossbar array the experimentally derived weights are compared to the ideal numeric weights. The numeric analyses simulate the perfect EC-RAM, i.e. no read/write noise and no nonlinearity. Clipping is also disabled for the numeric analysis which removes all restrictions on maximum and minimum weight values allowing operation across the full range. The write noise and nonlinearity that is used in the experimental analyses is directly derived from the data shown in Figure $2 \mathrm{e}$. The update value (conductance) consists of the average update step $\Delta \mathrm{G}_{0}$ (at a specific conductance state where CDF $=0.5$ ) plus the contribution of the write noise which is randomly sampled from the cumulative distribution function (CDF). The nonlinearity of the device is included as well since the average $\left(\Delta G_{0}\right)$ changes over the conductance domain. In this way, the measurement data accounts for both write noise and nonlinearity. Read noise is not taken into consideration as this is independent of the EC-RAM performance. 


\section{Reference}

[S1] Komáromy, D. et al. Self-Assembly Can Direct Dynamic Covalent Bond Formation toward Diversity or Specificity J. Am. Chem. Soc. 139, 6234-6241 (2017).

[S2] Higginbotham, H. F. et al. New brightly coloured, water soluble, core-substituted naphthalene diimides for biophysical applications Dyes and Pigments 112, 290 (2015).

[S3] Agarwal, S. et al. Resistive Memory Device Requirements for a Neural Algorithm Accelerator. in International Joint Conference on Neural Networks (2016).

[S4] van de Burgt, Y. et al. A non-volatile organic electrochemical device as a low-voltage artificial synapse for neuromorphic computing. Nat. Mater. 16, 414-418 (2017).

[S5] CrossSim. https://cross-sim.sandia.gov/

[S6] K. Bache and M. Lichman. UCI machine learning repository [Online]. Available:

http://archive.ics.uci.edu/ml/

[S7] Y. LeCun, C. Cortes, and C. J. Burges. The MNIST database of handwritten digits

[Online]. Available: http://yann.lecun.com/exdb/mnist 